\title{
1 Systems-based approach for optimization of a scalable bacterial ST mapping
}

\section{2 assembly-free algorithm}

3

4 Natasha Pavlovikj ${ }^{1 \uparrow}$, Joao Carlos Gomes-Neto ${ }^{2,3 \pi}$, Jitender S. Deogun ${ }^{1}$, Andrew

5 K. Benson ${ }^{2,3 *}$

6

$7{ }^{1}$ Department of Computer Science and Engineering, University of Nebraska-

8 Lincoln, Lincoln, Nebraska, United States of America

$9{ }^{2}$ Department of Food Science and Technology, University of Nebraska-Lincoln,

10 Lincoln, Nebraska, United States of America

$11{ }^{3}$ Nebraska Food for Health Center, University of Nebraska-Lincoln, Lincoln,

12 Nebraska, United States of America

13

$14 *$ Corresponding Author:

15 E-mail: abenson1@unl.edu (AKB)

16

17 T These authors contributed equally to this work. 


\section{Abstract}

25 Epidemiological surveillance of bacterial pathogens requires real-time data

26 analysis with a fast turn-around, while aiming at generating two main outcomes:

27 1) Species level identification; and 2) Variant mapping at different levels of

28 genotypic resolution for population-based tracking, in addition to predicting traits

29 such as antimicrobial resistance (AMR). With the recent advances and continual

30 dissemination of whole-genome sequencing technologies, large-scale population-

31 based genotyping of bacterial pathogens has become possible. Since bacterial

32 populations often present a high degree of clonality in the genomic backbone (i.e.,

33 low genetic diversity), the choice of genotyping scheme can even facilitate the

34 understanding of ancestral relationships and can be used for prediction of co-

35 inherited traits such as AMR. Multi-locus sequence typing (MLST) fits that

36 purpose and can identify sequence types (ST) based on seven ubiquitous genome-

37 scattered loci that aid in genotyping isolates beneath the species level. ST-based

38 mapping also standardizes genotyping across laboratories and is used by

39 laboratories worldwide. However, algorithms for inferring ST from Illumina

40 paired-end sequencing data typically rely on genome assembly prior to

41 classification. Genome assembly is computationally intensive and is a bottleneck

42 for speed and scalability, which are important aspects of genomic epidemiology.

43 The stringMLST program uses an assembly-free, kmer-based algorithm for

44 inferring STs, which can overcome the speed and scalability bottlenecks. Here we

45 have systematically studied the accuracy and scalability of stringMLST relative to

46 the standard MLST program across a wide array of phylogenetically divergent 
47 Public Health-relevant bacterial pathogens. Our data shows that optimal kmer

48 length for stringMLST is species-specific and that genome-intrinsic and -extrinsic

49 features can affect performance and accuracy of the program. While suitable

50 parameters could be identified for most organisms, there were a few instances

51 where this program may not be directly deployable in its current format. More

52 importantly, we integrated stringMLST into our freely available and scalable

53 hierarchical-based population genomics platform, ProkEvo, and further

54 demonstrated how the implementation facilitates automated, reproducible

55 bacterial population analysis. The ProkEvo implementation provides a rapidly

56 deployable genomic epidemiology tool for ST mapping along with other pan-

57 genomic data mining strategies, while providing specific guidance on how to

58 optimize stringMLST performance for a wide variety of bacterial pathogens.

\section{Introduction}

61 Modern epidemiological investigation of bacterial pathogens relies on rapid

62 genomic characterization of new isolates routinely received by Public Health

63 laboratories, along with bioinformatics programs for classification/comparison of

64 genomic data from new isolates to existing data from many thousands of isolates

$65[1][2]$. While surveillance, source-tracking, and attribution are primary goals for

66 use of whole-genome sequencing (WGS) data by Public Health agencies, there is

67 growing interest in using the WGS data from thousands to hundreds of thousands

68 of isolates of a given pathogen to study hierarchical genotypic classification at

69 different levels of resolution, and prediction of population-specific traits 
70 contributing to virulence, ecological fitness, or antimicrobial resistance (AMR)

$71[3]$.

72 Evolutionarily-related bacterial populations (e.g., species and subtypes of a

73 species) share common genotypic backbones and the degree of clonality of a

74 population provides a context for studying co-inheritance of core- and accessory-

75 genes $[4][5][6][7]$. Core loci are shared by at least $99 \%$ of the genomes, whereas

76 accessory loci represent a sparse ensemble that are not shared by all variants of a

77 population, but jointly, the core and accessory genomic content form a given

78 species forms its pan-genome [66]. Population genetic analysis of bacterial

79 species using multi-locus methods have shown that multiple types of genotyping

80 schemes can provide sufficient resolution for classifying populations based on

81 genotypes beneath the species level [8][22][79][80][81], and the genetic

82 relationships between different populations reveal the pattern of population

83 diversification and structuring. This optimal level of genotypic resolution can be

84 considered an informative genotypic unit that facilitates both ecological and

85 epidemiological inquiries [57][82][83].

86 Multi-locus sequence typing (MLST) is a well-established and widely used

87 genotyping technique that classifies bacterial genomes into sequence types (ST)

88 [8][81]. ST classification based on traditional MLST is usually inferred from

89 seven loci that are found ubiquitously in the species and are scattered around the

90 genome. Highly curated, species-specific databases of allelic variation at MLST

91 loci and distributions of STs are publicly available [27][30][67]. Historically,

92 sequences for MLST loci were generated from locus-specific polymerase chain 
93 reaction (PCR) assays, but now are typically inferred from assembled WGS data

$94[8][9][44]$.

95 ST-based classification provides useful and relevant genotypic units for

96 epidemiological surveillance, population genetic analysis, and evolutionary

97 inference. Relative to surveillance, ST-based genotyping standardizes the

98 nomenclature for intra- and inter-laboratorial diagnostics and epidemiological

99 inquiries worldwide [9][10][11]. With respect to evolutionary inference, isolates

100 sharing alleles at five or more loci are more likely to be ancestrally related and are

101 commonly classified as members of a clonal complex (i.e., group of STs that have

102 shared a common ancestor very recently) [8][9][28][44]. The ST and clonal

103 complexes provide a point of reference for genetic analysis (e.g., Linkage

104 Disequilibrium - LD) to track inheritance/co-variance of accessory loci among

105 different STs or clonal complexes. For example, LD analyses of pan-genomic

106 content can inform phenotypic predictions for inheritance of unique traits in an ST

107 related to virulence, environmental stability, or AMR [12][13][14].

108 Recent studies have shown that LD between specific sets of accessory genes

109 such as AMR genes is an intrinsic property of some STs of specific bacterial

110 pathogens, and rapid identification of a given ST from WGS data can quickly

111 provide accurate prediction of AMR profiles [14][15]. There are important

112 applications of this innovative strategy for treatment of patients and there is

113 growing interest in defining LD relationships of AMR genes and virulence-

114 associated genes with STs. A limiting factor in ST-based classification from WGS

115 data, however, is the dependence of on genome assembly, most often from 
116 Illumina-based short-read data [27][30][67]. This bottleneck can also hinder ST-

117 based surveillance efforts when many thousands of genomes are involved in a

118 real-time analysis $[16][21]$. One approach to overcome the computational

119 bottleneck is to use kmer-based ST classification directly from Illumina paired-

120 end raw reads (assembly-free). The stringMLST algorithm was recently

121 developed for this purpose and shown to provide computationally efficient,

122 assembly-free ST classifications [17]. However, stringMLST has not been

123 broadly evaluated across data from multiple Public Health-related bacterial

124 pathogenic species. Given the remarkable variation in the intrinsic features of

125 genomes from different species (e.g., compositional features, abundances of

126 repetitive sequences, mutation rates, and rates of recombination); it is likely that

127 parameters for assembly-free algorithms, such as stringMLST, will need to be

128 empirically optimized for genetically diverse species and even subtypes (e.g.,

129 serotypes) of pathogens.

130 To test this hypothesis, we systematically examined the performance of

131 stringMLST across a phylogenetically diverse set of bacterial pathogens that are

132 of primary interest to Public Health. Our systematic approach compared accuracy

133 of classifications using the standard MLST program vs. stringMLST at varying

134 kmer lengths across many thousands of genomes from 15 different pathogenic

135 species from three highly divergent phyla. Performance was first evaluated across

136 a broad spectrum of phylogenetic diversity using genomes from isolates of

137 Acinetobacter baumannii (Phylum: Proteobacteria), Clostridium difficile

138 (Phylum: Firmicutes), Enterococcus faecium (Phylum: Firmicutes), Escherichia 
139 coli (Phylum: Proteobacteria), Haemophilus influenzae (Phylum: Proteobacteria),

140 Helicobacter pylori (Phylum: Proteobacteria), Klebsiella pneumoniae (Phylum:

141 Proteobacteria), Mycobacterium tuberculosis (Phylum: Actinobacteria), Neisseria

142 gonorrhoeae (Phylum: Proteobacteria), Pseudomonas aeruginosa (Phylum:

143 Proteobacteria), Streptococcus pneumoniae (Phylum: Firmicutes), Campylobacter

144 jejuni (Phylum: Proteobacteria), Listeria monocytogenes (Phylum: Firmicutes),

145 Salmonella enterica (Phylum: Proteobacteria), and Staphylococcus aureus

146 (Phylum: Firmicutes). To evaluate performance within diverse populations of a

147 single species, we also measured performance of different kmer lengths across 23

148 of the most relevant serovars of Salmonella enterica subsp. enterica lineage I ( $S$.

149 enterica). Our results show that optimal performance of stringMLST can, for

150 some species, be achieved with a single kmer length. However, several different

151 bacterial species, and even different serovars of $S$. enterica, require species or

152 serovar-specific kmer lengths for accurate ST classifications. Based on these

153 findings, we further implemented stringMLST into the scalable ProkEvo platform

154 [21], which produces hierarchical genotypic classifications from raw WGS data,

155 and demonstrated how use of species-specific kmer settings for stringMLST

156 enhances computational performance of the platform.

$157 \quad[4][5][6][7][66][8][27][30][67][8][9][44][14][15][27][30][67][16][21][17]$

\section{Materials and methods}

159 This systems-based comparison between mlst and stringMLST was centered at

160 capturing their differences in computational and statistical performances, and was 
161 accomplished through the following steps: 1) Narrow-scope comparative analysis

162 across four phylogenetic distinct pathogens species; 2) Further examination of

163 algorithmic performance within a single ecologically diverse bacterial species;

164 and 3) Wide-scope comparison between phylogenetic divergent pathogenic

165 species with Public Health relevance and with databases available on pubMLST

166 (https://pubmlst.org/) for direct contrast between stringMLST and mlst.

167

168 Datasets used for narrow-scope analysis

169 WGS data from four major bacterial pathogens, including Campylobacter jejuni,

170 Listeria monocytogenes, Salmonella enterica subps. enterica lineage I ( $S$.

171 enterica) and Staphylococcus aureus, were selected to be used in this first part of

172 the study. Our basis for that choice was due to three a priori defined criteria: 1)

173 Select bacterial species from two main phylogenetic divergent Phyla: Firmicutes

174 (L. monocytogenes and S. aureus) and Proteobacteria (C.jejuni and S. enterica);

175 2) Select zoonotic pathogens that continually cause human illnesses worldwide

176 [18]; and 3) Consider their epidemiological relevance according to the Centers for

177 Disease Control and Prevention (CDC) [19]. Specifically for S. enterica, 20 of the

178 CDC most investigated serovars were represented in the dataset, which includes:

$179 S$. Agona, S. Anatum, S. Braenderup, S. Derby, S. Dublin, S. Enteritidis, S. Hadar,

$180 S$. Heidelberg, $S$. Infantis, $S$. Javiana, $S$. Johannesburg, $S$. Kentucky, $S$. Mbandaka,

$181 S$. Montevideo, $S$. Muenchen, $S$. Newport, $S$. Schwarzengrund, $S$. Senftenberg, $S$.

182 Thompson, and $S$. Typhimurium [20]. All publicly available raw paired-end

183 Illumina reads for these organisms were downloaded from NCBI using parallel- 
184 fastq-dump [58]. Genomes used for all analyses were randomly selected from a

185 previously downloaded samples of isolates containing C.jejuni $(\mathrm{n}=21,919$

186 genomes), L. monocytogenes ( $\mathrm{n}=19,633$ genomes), S. enterica $(\mathrm{n}=25,284$

187 genomes), and $S$. aureus ( $\mathrm{n}=11,990$ genomes) that were processed through the

188 computational platform ProkEvo [21]. Specifically, our study design was

189 comprised of random sampling of 600 genomes from each species, except for $S$.

190 enterica for which 600 genomes were randomly drawn per serovar (list of all 20

191 serovars is shown in S1 Table). For each species and all S. enterica serovars, all

$192 \sim 600$ genomes were randomly split into three independent batches, with $\sim 200$

193 genomes each. The batches were created to measure the degree of variation in

194 classification accuracy when comparing the two ST-based genotyping programs.

195 While for the majority of S. enterica serovars there were a total of 600 genomes

196 available, the total number of raw reads publicly available on NCBI and

197 ultimately used for the analyses for $S$. Agona, $S$. Derby, $S$. Johannesburg, $S$.

198 Mbandaka and S. Senftenberg was 565, 590, 534, 535 and 563 respectively. The

199 final total number of genomes used per species was C. jejuni $(\mathrm{n}=600), L$.

200 monocytogenes $(\mathrm{n}=600)$, S. enterica $(\mathrm{n}=11,787)$, and $S$. aureus $(\mathrm{n}=600)$. Text

201 file containing all genome NCBI-SRA identifications is available here,

202 https://figshare.com/articles/dataset/_/16735411. 


\section{Software tools}

205 mlst

206 mlst is a standard approach for scanning genome assemblies against traditional

207 PubMLST typing schemes [22]. The genome assemblies can be in

208 FASTA/GenBank/EMBL formats [22]. mlst (version 2.16.2) was installed using

209 Anaconda, a package and environment manager that supports maintaining and

210 installing various open-source conda packages [26]. mlst uses genome assemblies

211 as an input. In order to generate assemblies from the raw Illumina paired-end

212 reads, multiple pre-processing steps were performed. Quality trimming and

213 adapter clipping were performed using Trimmomatic [50], while FastQC was

214 used to check and verify the quality of the trimmed reads [51]. The paired-end

215 reads were assembled de novo into contigs using SPAdes with the default

216 parameters [52]. The quality of the assemblies was evaluated using QUAST [53].

217 The information obtained from QUAST was used to discard assemblies with 0 or

218 more than 300 contigs, or assemblies with N50 value of less than 25,000 [21].

219 Finally, the assemblies that passed the quality control were used with mlst, where

220 they are categorized into specific variants based on the allele combinations from

221 seven ubiquitous, house-keeping genes [22]. A list of the exact versions of the

222 bioinformatics tools used for generating assemblies for mlst are shown on S2

223 Table. We used mlst with the default options (e.g., mlst--legacy --scheme

$224<$ scheme $>--$ csv $<$ assembly.fasta $>><$ output $>$ ) and the following schemes:

225 “senterica" (for S. enterica), "campylobacter" (for C. jejuni), "Imonocytogenes"

226 (for L. monocytogenes), "saureus" (for S. aureus). The distribution of mlst comes 
227 with set of pre-downloaded ST schemes. More details about these MLST

228 schemes, such as the number of alleles in the seven genes and the number of ST

229 classifications available are shown on S3 Table. To obtain the ST classifications

230 of all datasets, mlst was run as part of the computational platform ProkEvo [21].

231 Additionally, a separate run of the mlst program was used to conduct a pairwise

232 comparison between the computational performance (runtime and memory usage)

233 of mlst and stringMLST. The used mlst script can be found here,

234 https://github.com/npavlovikj/MLST_stringMLST_analyses/blob/main/scripts/ml

235 st.submit.

\section{stringMLST}

238 stringMLST is an assembly- and alignment-free rapid tool for ST-based

239 classification of Illumina paired-end raw reads based on kmers [17]. For the

240 analyses performed in this paper, we used stringMLST version 0.6.3. stringMLST

241 was installed using Anaconda [26]. The first step of using stringMLST was to

242 download the respective MLST scheme from PubMLST. In order to do this, a

243 species name and a kmer length were needed. The default kmer length used and

244 suggested by the developers of stringMLST for reads with lengths between 55 and

245150 base pairs or nucleotides is 35 (common read length for Illumina paired-end

246 reads) [17]. We used stringMLST with the default options (e.g., stringMLST.py --

247 getMLST --species $=<$ species_name $>-P<$ output_prefix $>-k<k m e r>)$ and the

248 following species names, "Salmonella enterica", "Campylobacter jejuni",

249 "Listeria monocytogenes", "Staphylococcus aureus", and kmer lengths of 10, 20, 
$25030,35,45,55,65,70,80,90$ independently

251 (https://github.com/npavlovikj/MLST_stringMLST_analyses/blob/main/scripts/str

252 ingMLST_dbs.submit). More details about the downloaded MLST schemes, such

253 as the number of alleles in the seven genes and the number of ST classifications

254 available are shown on S3 Table. After the MLST scheme was downloaded and

255 prepared, the final step was to run "stringMLT.py -predict" for the ST

256 classification. For this, we ran stringMLST with the databases a priori created and

257 the respective paired-end raw reads and kmer lengths of 10, 20, 30, 35, 45, 55, 65,

$25870,80,90$ independently (e.g., stringMLST.py --predict -d

$259<$ directory_raw_reads $>-p-r-t-x-P<$ database_prefix $>-k<k m e r>-o$

$260<$ output $>$ )

261 (https://github.com/npavlovikj/MLST_stringMLST_analyses/blob/main/scripts/str

262 ingMLST.submit). Our choice of using an increasing gradient of kmer lengths

263 was to evaluate whether the kmer length parameter could be optimized to enhance

264 ST-based classification accuracy across bacterial species. Lastly, stringMLST was

265 also integrated as part of the computational platform ProkEvo for a rapid ST-

266 based genotyping as part of a hierarchical genotypic scheme [21][57]. This

267 implementation can be found here,

268 https://github.com/npavlovikj/MLST_stringMLST_analyses/tree/main/Prokevo_st 269 ringMLST.

270

271 ProkEvo-based MLST classifications 
272 In order to compare the ST-based classification accuracy and conduct other

273 statistical analysis (e.g., identifying major contributing factors influencing ST-

274 based classifications) between mlst version 2.16.2 (assembly-dependent) and

275 stringMLST version 0.6.3 (assembly-independent), all initial ST calls for all

276 selected genomes, across all four species (C.jejuni, L. monocytogenes, S. aureus

277 and $S$. Typhimurium), were done using mlst [22] through the computational

278 platform ProkEvo [21]. In brief, ProkEvo uses bacterial Illumina raw paired-end

279 sequences as an input, and the following steps are sequentially done prior to ST-

280 based genotyping using mlst: Trimmomatic for sequence trimming [50], FastQC

281 for quality control of the trimmed reads [51], SPAdes for de novo genome

282 assembly [52], and QUAST for quality assessment of the genome assemblies

283 [53]. More information on how to install and use ProkEvo for hierarchical

284 bacterial population genomic analyses can be found here,

285 https://github.com/npavlovikj/prokevo.

\section{Genome-intrinsic and -extrinsic factors that can}

\section{8 influence algorithmic performance}

289 Both genome-intrinsic and -extrinsic factors were considered to determine their

290 contribution on the accuracy of ST classifications when comparing mlst vs.

291 stringMLST. The genome-intrinsic variables considered in these analyses were:

292 number of contigs per genome, total number of nucleotides per genome (genome

293 length), GC\% content per genome, and dinucleotide composition of genomes. The 
294 number of contigs per genome, as well as the genome length, were calculated

295 using the assembled contigs from SPAdes [52]. The number of contigs was

296 calculated for each genome using the Linux "grep" utility (e.g., grep "> "

297 assembly.fasta $\mid w c-l)$. The total number of nucleotides per genome was

298 calculated using the "getlengths" function from the AMOS package [54]. For this

299 analysis, we used AMOS v3.1. "getlengths" provides the length for each contig,

300 and a custom Bash script was used to summarize these values per genome. The

301 GC\% content was calculated using the program FastQC [51]. FastQC is used to

302 check and verify the quality of the raw Illumina paired-end raw reads. With each

303 pair of raw reads from all datasets, FastQC v0.11 was used. One of the statistics

304 checked for read quality is GC\% and this value was extracted with custom Bash

305 script from the file "fastqc_data.txt" once the FastQC output was generated. Since

306 FastQC outputs the GC\% per read, the average of both reads was calculated as the

307 final read GC\%. The dinucleotide composition of the genomes was calculated

308 with the function "compseq" from the EMBOSS package [55]. "compseq"

309 calculates the frequency of words of a specific length (e.g., length is 2 in the case

310 of dinucleotides) from given input genome sequences. For these analyses we used

311 EMBOSS v6.6 with the command "compseq -word 2 -outfile <output>

312 assembly.fasta" for all datasets and genomes a priori assembled with SPAdes

313 [52]. Next, a customized Bash script was used to count the total number of

314 occurrences of each dinucleotide for each genome across all bacterial species.

315 Finally, all these outputs were merged per genome using custom Python script to

316 facilitate statistical analyses and data visualization. The used scripts can be found 
317 here,

318 https://github.com/npavlovikj/MLST_stringMLST_analyses/tree/main/scripts.

319 The genome-extrinsic variables used in the analyses presented in this paper

320 were the total count of unique STs per database and the total count of unique

321 alleles across all seven loci used for ST classification across all bacterial species.

322 These genome-extrinsic variables were extracted from the PubMLST databases

323 for both stringMLST and mlst using custom Bash scripts. While the first step of

324 stringMLST is to download the most up-to-date available MLST scheme from

325 PubMLST, the distributed version of mlst comes with set of pre-downloaded ST

326 and allelic schemes. For each MLST scheme, the mlst distribution has a separate

327 directory with 8 files - seven are ".tfa" files with the fasta sequences of the alleles

328 for each locus, and one file (e.g., senterica.txt) contains the ST information (i.e.,

329 the total number of STs mapped including their specific allelic composition across

330 all seven loci for that given species). To calculate the total number of unique STs,

331 we used the Linux utility "wc" with the text file with ST information (e.g., wc -l

332 senterica.txt). To calculate the total count of unique alleles across the seven loci,

333 the "grep" Linux utility was used with the seven ".tfa" files (e.g., grep "> * *.tfa|

$334 w c-l)$. All calculations were done per bacterial species. The downloaded MLST

335 scheme with stringMLST is in a separate directory for each organism and used

336 kmer length. This directory had 12 files - seven are ".tfa" files with fasta

337 sequences for all alleles across all seven loci, and one file has the ST profiles

338 (e.g., Salmonella_enterica_profile.txt), while the remaining files contained

339 information about the extracted kmers and additional config and log information. 
340 Similarly, the total number of unique STs for stringMLST was counted using the

341 Linux utility "wc" with the text file with ST profile information (e.g., $w c-l$

342 Salmonella_enterica_profile.txt), and the total count of unique alleles per loci was

343 extracted using the "grep" Linux utility with the seven ".tfa" files (e.g., grep "> "

$344 *$.tfa $\mid w c-l)$. Similarly, all ST and allelic counts were carried out per bacterial

345 species. With stringMLST, the MLST schemes are downloaded and prepared

346 separately for each different kmer length used. However, the kmer length did not

347 affect the number of STs and unique alleles per organism. Thus, these values

348 remained the same across organisms and kmer lengths for stringMLST.

\section{Kmer-based distribution across ST programs}

351 In order to assess the potential impact of random mapping or occurrence of kmers

352 of different lengths across different bacterial species, we randomly chose 100 raw

353 Illumina paired-end reads from the initial C.jejuni, L. monocytogenes, S. aureus

354 and $S$. Typhimurium (major representative zoonotic serovar of S. enterica)

355 isolates. For each read, we extracted all unique kmers of length 10, 20, 30, 35, 45,

$35655,65,70,80$ and 90 respectively, and counted their occurrence in the

357 corresponding raw reads. This was done using DSK v2.2.0 [56]

358 (https://github.com/npavlovikj/MLST_stringMLST_analyses/blob/main/scripts/ds

359 k.submit). Next, the total number of kmer frequency was summarized per

360 organism and kmer length, and the mean value was calculated to examine the

361 distribution of different kmers across the raw reads. For each database created

362 with stringMLST, a file with the kmer frequency for the used ST scheme was 
363 generated. Using the kmers generated from the raw reads and the stringMLST

364 database, a relative frequency of the common kmers was calculated (calculated as

365 a ratio between the common kmers and the unique kmers from all the kmers

366 generated between the raw reads and the stringMLST database, e.g.,

367 (common_kmers/unique_total_observations)*100). The code used for this can be

368 found in our GitHub repository

369 (https://github.com/npavlovikj/MLST_stringMLST_analyses/tree/main/figures_c 370 ode).

371

372 Agreement in ST classification between programs

373 In order to assess the overall accuracy of stringMLST compared to the standard

374 mlst approach for ST calls, a percentage of agreement in ST classification was

375 calculated. For this, the initial dataset composed of 600 genomes from either $C$.

376 jejuni, or L. monocytogenes, or S. aureus was selected, in addition to a total of

37711,787 genomes across twenty zoonotic serovars of $S$. enterica $(\sim 600$ genomes

378 per serovar, S1 Table). The program stringMLST was run with increasing kmer

379 lengths ranging from 10 to 90 nucleotides. If both stringMLST and mlst produced

380 identical ST calls, either "good" or "bad" ones, the call was a match. A "good"

381 and "bad" call represent ST with a number or a missing/blank value, respectively.

382 The remaining combinations were classified as a mismatch. Next, the percentage

383 of agreement (concordance) was calculated with custom R base script

384 (https://github.com/npavlovikj/MLST_stringMLST_analyses/tree/main/figures_c 385 ode). 


\section{Computational platforms}

388 All computational analyses performed for this paper were done on Crane - one of

389 the high-performance computing clusters at the University of Nebraska-Lincoln

390 Holland Computing Center [23]. Crane is Linux cluster, having 548 Intel Xeon

391 nodes with RAM ranging from 64 GB to 1.5 TB. The scalability of ProkEvo with

392 stringMLST was tested on the Open Science Grid (OSG), a distributed, high-

393 throughput computational platform for large-scale scientific research [24][25].

394 OSG is a national consortium of more than 100 academic institutions and

395 laboratories that provide storage and tens of thousands of resources to OSG users.

396 These sites share their idle resources via OSG for opportunistic usage. The OSG

397 resources are Linux-based, and due to the different sites involved, the hardware

398 specifications of the resources are different and vary.

399

400 Computational performance

401 To evaluate the computational performance of stringMLST in comparison to the

402 mlst program, we assessed the runtime and memory usage of both programs. For

403 this, we chose four different datasets, C. jejuni, L. monocytogenes, S. aureus and

$404 S$. Typhimurium (major representative zoonotic serovar of $S$. enterica), with three

405 different batches of 200 genomes each, with a total of 600 genomes each. We ran

406 mlst with all required steps, such as quality trimming and adapter clipping, de

407 novo assembly, and assembly discarding on each dataset (see Section Software

408 tools: mlst for more detailed description). Separately, we ran stringMLST with a 
409 range of 10 different kmer lengths $(10,20,30,35,45,55,65,70,80,90)$ on each

410 dataset. For each organism, the runtime was calculated as an average of all 200

411 genomes per batch. In general, the runtime depends on multiple factors, such as

412 the specification and capabilities of the used computational platform. Since the

413 runtime can vary depending on these various factors, average statistics were used

414 to show the central tendency of the runtime when comparing stringMLST vs.

415 mlst. The runtime was calculated using the "date" command integrated in the

416 Unix operating systems (e.g., $t={ }^{\prime}$ date $+\% s^{`} ;$ mlst --legacy --scheme senterica --

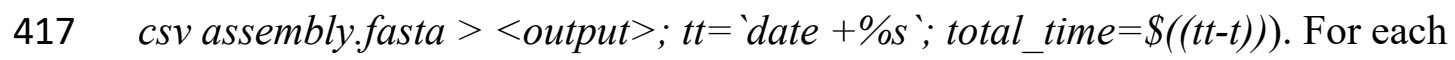

418 organism, the memory was calculated as the maximum memory recorded from all

419200 genomes per batch, since all genomes were analyzed separately and

420 concurrently. In the case of mlst, the recorded memory was the maximum

421 memory of all the steps ran prior to mlst, such as trimming, de novo assembly,

422 quality checking, filtering, and ST typing. The memory used for these steps

423 considerably varies from a few MBs to a few GBs (e.g., filtering vs. de novo

424 assembly), and since the memory is a physical limitation of the computational

425 platform, the maximum used memory was calculated for each organism and

426 batch. The memory used was calculated using the "cgget" command that tracks

427 various parameters from the Linux Control Groups (cgroups) per running job

428 (e.g., mlst--legacy --scheme senterica --csv assembly.fasta $><$ output >; $r={ }^{\prime}$ cgget

429 -r memory.usage_in_bytes /slurm/uid_\$\{UID\}/job_\$\{SLURM_JOBID $\} /$;

$430 \quad m e m=$ 'echo $\$ r \mid$ awk $-F:$ ' $^{\prime}$ print $\left.\left.\$ 3\right\}^{\prime \prime}\right)$. 


\section{Incorporating stringMLST in ProkEvo}

433 ProkEvo is a freely available and scalable computational platform capable of

434 facilitating bacterial population genomics analyses while combining various

435 independent algorithms in a portable pipeline [21]. One of the advantages of

436 ProkEvo is its ability to facilitate the addition and removal of new steps and

437 programs without disrupting its workflow. For instance, more details about adding

438 new programs to ProkEvo are given here

439 https://github.com/npavlovikj/ProkEvo/wiki/4.1.-Add-new-bioinformatics-tool-

440 to-ProkEvo. By following these instructions, we were able to successfully add

441 stringMLST to the current ProkEvo platform. The ultimate description of how

442 stringMLST was integrated into ProkEvo can be found here,

443 https://github.com/npavlovikj/MLST_stringMLST_analyses/tree/main/Prokevo_st 444 ringMLST.

446 Comparison between mlst and stringMLST performance

\section{7 using ProkEvo}

448 In order to compare the performance/accuracy of MLST and stringMLST as part

449 of the ProkEvo platform, two subsets of the C. jejuni, L. monocytogenes, $S$.

450 Typhimurium and S. aureus datasets used in this paper were selected. One subset

451 was composed of 100 randomly selected genomes, while the second one

452 contained 1,000. The subsets were randomly selected from the original datasets

453 used in this paper. As part of ProkEvo, stringMLST was run with the default kmer 
454 length of 35. The ProkEvo workflows with mlst and stringMLST and the two

455 datasets were individually run on Crane - one of the high-performance computing

456 clusters at the Holland Computing Center. Once the four workflows finished, the

457 performance of ProkEvo with mlst and stringMLST and the datasets with 100 and

458 1,000 genomes, respectively, was compared using: i) the total running time; ii) the

459 percentage of non-classified STs; and iii) the percentage of agreement between

460 programs. Since ProkEvo is an automated platform, a list of NCBI-SRA

461 identifications was provided with the ProkEvo implementations with both mlst

462 and stringMLST. In brief, ProkEvo manages all the dependencies and

463 intermediate steps, and produces the final ST classification as an output.

464

465 stringMLST-based kmer length optimization across

466 phylogenetic divergent bacterial pathogens

467 To identify the optimal species-specific kmer length that minimizes the frequency

468 of ST miscalls, we ran stringMLST with a range of different kmer lengths across

469 phylogenetic divergent pathogenic species. First, we chose twenty-three $S$.

470 enterica serovars ( $S$. Agona, $S$. Anatum, $S$. Braenderup, $S$. Derby, $S$. Dublin, $S$.

471 Enteritidis, $S$. Hadar, $S$. Heidelberg, $S$. Infantis, $S$. Javiana, $S$. Johannesburg, $S$.

472 Kentucky, S. Mbandaka, S. Montevideo, S. Muenchen, S. Newport, $S$.

473 Oranienburg, $S$. Poona, $S$. Saintpaul, $S$. Schwarzengrund, S. Senftenberg, $S$.

474 Thompson, $S$. Typhimurium), and for each dataset we randomly selected 100

475 paired-end Illumina reads from NCBI-SRA. Second, for each dataset we ran mlst

476 and stringMLST with kmer lengths ranging from 20, 30, 35, 40, 45, 50, 55, 60, 
$47765,70,80,90$. The kmer length of 10 was excluded due to its poor performance in

478 previous analyses. Additionally, we use data from fourteen pathogens with Public

479 Health relevance and with available MLST schemes, to widen the scope of the

480 analysis and assess the necessity of fine-tunning the kmer length on a more

481 broadly selected collection of species. In particular, we chose the following

482 pathogens: Acinetobacter baumannii, Clostridioides difficile, Enterococcus

483 faecium, Escherichia coli, Haemophilus influenzae, Helicobacter pylori,

484 Klebsiella pneumoniae, Mycobacterium tuberculosis, Neisseria gonorrhoeae,

485 Pseudomonas aeruginosa, Streptococcus pneumoniae, Campylobacter jejuni,

486 Listeria monocytogenes, and Staphylococcus aureus. For each pathogen, we

487 randomly selected and downloaded 1,000 paired-end reads from NCBI-SRA and

488 processed these reads separately with mlst and stringMLST. stringMLST was run

489 with kmer lengths ranging from 20, 30, 35, 45, 55, 65, 70, 80, 90 and different

490 schemes for the different pathogens. Similar to the S. enterica datasets, the kmer

491 length of 10 was excluded from the analysis.

492 Across all datasets, the percentage of ST miscalls was calculated for

493 stringMLST for each kmer length, whereby miscalls were defined as "bad" ST

494 calls - calls with a missing or blank values. Next, for each dataset, the kmer length

495 that equated with the lowest percentage of ST miscalls was recorded. For some

496 datasets, multiple kmer lengths generated an identical lowest percentage for ST

497 miscalls. In this case, we applied a two-folded approach to select the most optimal

498 kmer length: 1) if kmer of length 35 was part of the array of kmer lengths that

499 showed the most optimal results, we recorded kmer 35 as the optimal kmer length 
500 since that is the default and recommended value for stringMLST (parsimonious

501 approach); or 2) if kmer of length 35 was not part of the kmer lengths that showed

502 the most optimal results, we recorded the kmer with the highest value as the most

503 optimal one, since in general our analysis showed that longer kmers consumed

504 less computational resources and speed up the entire analysis. Ultimately, the

505 optimal kmer length and the percentage of ST miscalls were visualized onto a

506 core-genome phylogeny generated for all twenty-three $S$. enterica serovars, as

507 well as for all fourteen pathogens including all twenty-three S. enterica serovars

508 which jointly totaled fifteen pathogens (total of 37 genomes, one per species

509 including one per serovar of S. enterica, were used to construct the 16S rRNA-

510 based phylogeny for visualization purposes). The core-genome alignment for the

511 twenty-three S. enterica serovars was generated using Roary with this set of

512 parameters, “roary -s -e --mafft -p 8 -cd 99 -i 95 ./prokka_output/*.gff-f

513 roary_output" and the phylogenetic tree was produced using FastTree [76]. The

514 phylogenetic tree for the fifteen pathogens was generated using CLUSTALW

515 (https://www.genome.jp/tools-bin/clustalw) with the 16S rRNA sequences of the

516 selected 37 genomes generated by Prokka [78]. All phylogeny-based

517 visualizations were done using iTOL [77], and the recorded and overlaid statistics

518 were extracted with custom R scripts

519 (https://github.com/npavlovikj/MLST_stringMLST_analyses/blob/main/figures_c

520 ode/figures_code.Rmd).

521 In addition to calculating the percentage of ST miscalls for different kmer

522 lengths with stringMLST, for each dataset we calculated the percentage of 
523 agreement (concordance) between mlst and stringMLST on ST calls ("good" or

524 "bad"), as previously described here. Of note, when the stringMLST and mlst

525 results were combined, the number of returned ST calls was not always 1,000 (the

526 original size of the used datasets). If 1,000 reads are used with stringMLST,

527 stringMLST generates ST calls for all 1,000 reads. On the other hand, when using

528 mlst, a set of steps are used before mlst, including filtering, and a fraction of

529 assemblies were disregarded due to poor quality. Thus, only genome sequences

530 that passed through the mlst program and yielded a "good" or "bad" call were

531 ultimately used to compare with stringMLST. The number of raw reads for each

532 dataset, as well as the number of final reads from mlst used for these analyses are

533 shown on S4 Table.

534

535 Statistical analyses

536 To compare the overall performance and accuracy of mlst vs. stringMLST on ST-

537 based classifications, the following statistics were used across all bacterial species

538 datasets: 1) ST richness; 2) Simpson's D index (1 $-D)$ of diversity using ST

539 counts as input data; 3) Proportion of non-classified STs (missing values or blank

540 calls); and 4) Standard deviation of the proportion of non-classified STs. These

541 statistics were calculated to evaluate the algorithmic performance on ST-based

542 classification accuracy within and between bacterial species selected to be used in

543 the narrow scope analysis (C. jejuni, S. aureus, L. monocytogenes, and $S$.

544 enterica). ST richness was calculated by identifying the number of distinct STs

545 present in each species. The Simpson's D index of diversity (1- D) was used to 
546 calculate the degree of genotypic diversity across species, using the diversity()

547 function available in the vegan (version 2.5-6) R library [29]. The proportion of

548 non-classified STs was calculated using the counts of isolates or genomes that

549 were not assigned a ST number after each run of either mlst or stringMLST. The

550 standard deviation of the proportion of non-classified STs was calculated using

551 the $\operatorname{sd}()$ function which is derived from an unbiased estimate of the sample

552 variance corrected by $n-1$ ( $n$ for number of observations). The frequency of

553 genomes used for all analyses was calculated per batch and program across all

554 species, including across serovars for S. enterica. The relative frequency of the

555 most dominant ST lineages was also assessed across bacterial species.

556 PERMANOVA univariate or multivariate models were used to assess the

557 significance $(p<0.05)$ and the degree of association between the genome-intrinsic

558 and -extrinsic factors with the following dependent variables: ST richness,

559 Simpson's D index of diversity, or proportion of non-classified STs. Statistical

560 models were built for each of the dependent variables separately. Multivariate

561 models included either the combination of bacterial species and program, or

562 serovars in the case of S. enterica and program. These multivariate models were

563 stated to calculate the main and synergistic effects of the explanatory variables

564 (e.g., species*program or serovar*program). Univariate models were also

565 assessed for each of the dependent variables, using one of the following

566 independent/explanatory variables: 1) Genome-intrinsic variables: median number

567 of contigs, mean of the total count of nucleotides per genome, mean of the

568 average $\mathrm{GC} \%$ content per genome, standard deviation of the number of contigs, 
569 standard deviation of the total count of nucleotides per genome, and standard

570 deviation of the average GC\% content per genome; 2) Genome-extrinsic

571 variables: species, serovar of S. enterica, program (mlst vs. stringMLST with

572 kmer lengths of $10,20,30,35,45,55,65,70,80,90)$, mean of the total count of

573 unique STs per program, mean of the total count of unique alleles across all genes

574 per program, and the Simpson's D index of diversity per species. Statistical

575 significance and strength of association between the dependent and independent

576 variables were measured with $p$-values $(p<0.05)$ and $R$-squared, respectively. In

577 the case of contig size (median), total number of nucleotides per genome (mean),

578 and GC\% content per genome, summary statistic values (median or mean) were

579 calculated grouped by species and batch (there was a total of three batches per

580 bacterial species or serovar). For the total count of STs and total number of alleles

581 in the database, summary statistic values (mean) were calculated grouped by

582 species, batch, and program. Lastly, the standard deviation of number of contigs,

583 total count of nucleotides per genome, or GC\% content per genome were

584 calculated grouped by species. PERMANOVA models were run using the

585 adonis() function with 1,000 permutations using the vegan (version 2.5-6) $\mathrm{R}$

586 library [29]. Principal component analysis (PCA) was used to analyze the

587 dinucleotide distribution across species and across serovars for S. enterica with

588 two dimensions using the $\operatorname{prcomp}()$ function. The PCA calculations and the

589 selection of the number of PCs were done using the factoextra (version 1.0.7)

590 library. Bar-plots, box-and-whiskers plots, and bivariate/trivariate scatter plots

591 were used to assess and visualize the distribution and associations within and 
592 between dependent and independent/explanatory variables. The R software

593 (version 4.0.3) and R libraries such as Tidyverse (version 1.3.0) were used to

594 conduct all statistical analyses, and all R scripts are available here

595 (https://github.com/npavlovikj/MLST_stringMLST_analyses/tree/main/figures_c

596 ode). Data quality control was achieved with $\mathrm{R}$ base functions, in addition to the

597 following packages: skimr (version 2.1.3) and visdat (version 0.5.3). Graphical

598 visualizations were achieved using ggplot2 (version 3.3.2), GGally (version

599 2.1.2), and plotly (version 4.9.4.1). R code integrity was checked using the

600 assertive (version 0.3-6) package.

601

602 Results

603 The computational and analytical approaches used in this paper are shown on Fig

604 1. Our analytical approach was sub-divided into a narrow- and wide-scope

605 analysis aiming at accomplishing two goals: 1) Comparing the computational

606 performance and accuracy of MLST vs. stringMLST; 2) Optimizing the use of

607 stringMLST for a wide range of bacterial species; and 3) Implementing

608 stringMLST as part of the ProkEvo computational genomics platform. We

609 initially used publicly available raw Illumina paired-end sequence data from $C$.

610 jejuni, L. monocytogenes, S. enterica and S. aureus, to run stringMLST and mlst

611 independently in order to compare the accuracy in ST-based classifications and

612 assess the computational needs and performance in the overall analysis (narrow-

613 scope step). For this narrow-scope analysis, we performed a detailed comparative 
614 analysis between these two programs including: i) analyses of computational

615 performance and resources needed (e.g., average runtime per genome and

616 maximum memory needed to analyze all genomes), and ii) statistical analyses to

617 determine the accuracy of classifications (e.g., ST richness, Simpson's D index of

618 ST-based diversity, proportion of miscalls, and percentage of agreement or

619 concordance between programs). For the wide-scope step of the analysis, we

620 systematically evaluated the accuracy and concordance between mlst and

621 stringMLST across a broader array of phylogenetic divergent pathogens with

622 direct implication for Public Health (A. baumannii, C. difficile, E. faecium, E.

623 coli, H. influenzae, H. pylori, K. pneumoniae, M. tuberculosis, N. gonorrhoeae, $P$.

624 aeruginosa, S. pneumoniae, C. jejuni, L. monocytogenes, S. enterica and S.

625 aureus). Combined with the intra-species analysis done across 23 serovars of $S$.

626 enterica, our assessment aimed at revealing the optimized kmer length to be used

627 with stringMLST in order to: i) minimize the percentage of ST miscalls, and ii)

628 maximize the use of computational resources by speeding up the analysis. Lastly,

629 we provided an implementation of stringMLST within ProkEvo - a freely

630 available and scalable computational platform that facilitates hierarchical

631 genotyping of bacterial populations including pan-genomic mapping [21].

632

\section{Computational performance}

634 The computational performance between stringMLST and mlst was measured

635 using two metrics: 1) The average computational runtime per genome; and 2) The

636 maximum memory used per dataset. The average runtime in minutes per genome 
637 per batch between mlst and stringMLST with different kmer lengths, for C. jejuni,

638 L. monocytogenes, S. aureus, and $S$. Typhimurium (major representative of $S$.

639 enterica), is shown on Fig 2. While the runtime of mlst varies between 20 and 80

640 minutes per genome depending on the dataset used, all stringMLST runs with

641 different kmers finished within a few minutes (ranging from $\sim 1-16$ minutes when

642 kmer 10 was included and 1-5 minutes when kmer 10 was excluded). Apart from

643 stringMLST with kmer 10, all other kmer lengths showed a uniform runtime. The

644 longer runtime observed with kmer 10 can be partially explained by the higher

645 number of kmers that were generated and used for mapping (S1 Fig). The

646 obtained results show that ST-based classifications are accomplished considerably

647 more rapidly when carried out using stringMLST compared to the standard MLST

648 program.

649 Additionally, a comparison of maximum memory used when both stringMLST

650 and mlst were run for C. jejuni, L. monocytogenes, S. aureus, and S. Typhimurium

651 (major representative of S. enterica) is shown on S2 Fig. Across all species, the

652 range of maximum memory usage for mlst and stringMLST (across all kmers)

653 was $\sim 2-16 \mathrm{GBs}$ and $\sim 3-30 \mathrm{GBs}$ respectively. Although the memory used across

654 datasets is variable, none of the analyses we ran exceeded 30GBs of RAM. Since

655 most high-performance computers can consistently provide resources from 32GBs

656 to a few TBs of RAM, the memory available should not be considered a

657 bottleneck for running either program.

658 


\section{Factors that can influence $\mathbf{S T}$-based classification}

660 First, we describe the characteristics and composition of the data utilized for

661 comparison between programs regarding ST-based classification in the narrow-

662 scope approach (utilization of fewer phylogenetic diverse pathogen datasets). The

663 frequency of genomes utilized per species and across programs is shown on S3A-

664 D Fig. The frequency of S. enterica genomes was higher than other species

665 because an equal sample of $\sim 600$ genomes was taken from 20 representative

666 zoonotic serovars (S4A-D Fig). An assessment of the proportion of the most

667 dominant STs across species (proportion $\geq 2 \%$ - S5A-D Fig) or serovars of $S$.

668 enterica (proportion $\geq 15 \%$ - S4N Fig) initially revealed a similar ST-based

669 distribution across programs. Furthermore, genome-intrinsic and -extrinsic factors

670 that could potentially impact the mlst vs. stringMLST algorithmic comparison and

671 performance were a priori determined in the analysis. Among the genome-

672 intrinsic factors considered across species were the number of contigs per genome

673 (Fig 3A), the total number of nucleotides per genome (Fig 3B), GC\% content per

674 genome (Fig 3C), and the distribution and composition of dinucleotides per

675 species (Fig 3D and S3E-F Fig). Similarly, the distribution of the genome-

676 intrinsic factors was analyzed across all twenty serovars of S. enterica (S4G-L

677 Fig). A correlogram (pairwise correlation analysis) was also used to assess the

678 bivariate correlation (Pearson's correlation coefficient) across genome-intrinsic

679 variables, for either all four bacterial species (S3G Fig) or serovars across $S$.

680 enterica (S4M Fig). At large, the differences observed in the distribution of 
681 genomic-intrinsic variables were species driven, with a strong uniformity found

682

683

684

685

686

687

688

689

690

691

692

693

694

695

696 Assessing the contribution of genome-intrinsic and -

\section{7 extrinsic variables}

698 across serovars of $S$. enterica.

As for the genome-extrinsic variables, the total count of unique STs (for species - Fig 3E) and unique number of alleles across all seven loci (for species Fig 3F), and across all batches were selected as factors that could influence the comparative analysis between mlst and stringMLST. Similarly, the genomeextrinsic variables were analyzed across all twenty serovars of $S$. enterica (S4E-F Fig). Of note, database size differences (number of STs and alleles) may directly influence the number of miscalls since it is expected that the larger the database is, the more likely STs are to be classified, or to find a match, and not be miscalled [30]. Considering the differences in genome-intrinsic and -extrinsic variable distribution across species, such factors were further utilized for assessing their statistical contribution in the accuracy of ST-based classification between mlst and stringMLST.
In order to assess the statistical association and contribution of each genomicintrinsic and -extrinsic variable onto the accuracy of mlst vs. stringMLST on ST calls (narrow-scope analysis since it only included four bacterial species, $C$.

jejuni, S. aureus, L. monocytogenes, and S. enterica), the following dependent variables (outcomes) were used in the PERMANOVA models: 1) ST richness (Fig 4A); 2) Simpson's D index of ST diversity (Fig 4B); and 3) Proportion of 
704 non-classified STs (Fig 4C). Additionally, the standard deviation of the proportion

705 of non-classified STs was measured as an auxiliary metric for accuracy (Fig 4D).

706 At the species level, a multivariate model approach was used to examine the

707 interaction of species and program (mlst vs. stringMLST), whereas all remaining

708 analyses were done using univariate models containing each genome-intrinsic and

709 -extrinsic variable for all three outcomes (S6A-L Fig, S7A-K Fig, S8A-L Fig).

710 For each variable, the significance and strength of association were assessed by

711 jointly examining the $p$-value $(p<0.05)$ and $R$-squared, respectively. For both ST

712 richness (Fig 4A) and the Simpson's D index of diversity (Fig 4B), the difference

713 between species explained most of the variation with $\sim 98.3 \%$ and $\sim 99 \%$,

714 respectively. As expected, based on the phylogenetic divergence of the four

715 chosen pathogens, differences across species could largely be explained by

716 genome-intrinsic variables associated with genome composition, such as: GC\%

717 content ( $p \sim 0.0009, R$-squared $\sim 44 \%$ ) for ST richness, and the number of contigs

718 per genome ( $p \sim 0.0009, R$-squared $\sim 39.5 \%$ ) for the Simpson's D index of

719 diversity (Fig 4A-B). Notably, for both ST richness and the Simpson's D index of

720 diversity most of the differences between species could be explained by variation

721 in genome composition (Fig 4A-B). Not surprisingly, co-linearity was observed

722 between ST richness and the Simpson's D index of diversity across species (Fig

723 4A). In the case of the proportion of non-classified STs (ST miscalls) (Fig 4C),

724 most of the variation was explained by inter-species differences $(p \sim 0.0009, R$ -

725 squared $\sim 33 \%$ ), with the number of contigs per genome being the most important

726 genome-intrinsic contributing factor $(p \sim 0.0009, R$ - squared $\sim 27 \%)$. As for the 
727 kmer length parameter used by stringMLST, results for ST richness and the

728 Simpson's D index of diversity were uniform across all lengths (Fig 4A-B).

729 However, when examining the proportion of miscalls (Fig 4C) and the standard

730 deviation of that proportion (Fig 4D), the data pointed toward the optimal kmer

731 length being between 35 and 65 across all four species due to the intrinsic

732 variance within the S. enterica data (narrow scope analysis). Specifically, this

733 kmer length range was defined based on two criteria: i) minimization of the

734 proportion of miscalls; and ii) less variation (standard deviation) around the

735 average of ST-based miscalls. Of note, mlst demonstrated the highest proportion

736 of miscalls and standard deviation of that proportion for both L. monocytogenes

737 and $C$.jejuni (Fig 4C-D), and the kmer length 10 for stringMLST yielded very

738 low accuracy and null results for ST richness and Simpson's D index of diversity

739 (Fig 4A-D). Differences between species across ST richness, Simpson's D index

740 of diversity, and proportion of ST miscalls along with all genome-intrinsic and -

741 extrinsic variables across programs (mlst vs. stringMLST) were further examined

742 here (Fig 5A-D, S9A-O Fig). In general, differences in ST-based calls across

743 programs were largely influenced by the bacterial species dataset.

744 Given the complexity and diversity of the S. enterica population structure [12],

745 the stringMLST performance was analyzed across twenty zoonotic serovars

746 (S4O-R Fig), and resulted in a significant and predominant contribution of the

747 “serovar groupings" across all outcomes and PERMANOVA models (S10A-L

748 Fig, S11A-K Fig, S12A-L Fig): ST richness ( $p \sim 0.0009, R$-squared $\sim 75.4 \%)$,

749 Simpson's D index of diversity ( $p \sim 0.0009, R$-squared $\sim 88 \%$ ), and proportion of 
750 ST miscalls ( $p \sim 0.0009, R$ - squared $\sim 35.4 \%$ ). By assessing the distribution of the

751 model outcomes, along with PERMANOVA model results and bivariate

752 association between dependent and explanatory variables (S13A-R Fig), the

753 results recapitulated the species-level results with the optimal kmer length for

754 stringMLST being around 35 and 65, but also revealed the need to consider

755 difference across $S$. enterica serovars prior to implementation. Combined, these

756 accuracy-based results suggest that: i) stringMLST minimizes the ST miscalls

757 compared to mlst in a species-specific fashion, and by consequence the optimal

758 kmer length for stringMLST ranged from 35 to 65 overall; ii) the performance

759 and accuracy of stringMLST can vary across species and serovars of S. enterica

760 allowing for data-driven fine-tunning of the kmer length; and iii) the use of

761 sequence platform with longer reads, which would maximize the number of

762 contigs per genome, could directly alter both mlst and stringMLST accuracy in

763 ST calls across species.

764

\section{Concordance between programs}

766 Concordance between programs was calculated as the percentage of cases in

767 which outputs from both mlst vs. stringmlst agreed in the call ("good" or "bad").

768 Results demonstrating the percentage agreement in ST calls between mlst and

769 stringMLST with different kmer lengths are shown on Fig 6. Apart from kmer 10,

770 across all species, the percentage of agreement between mlst and stringMLST

771 varies between $\sim 81 \%$ and $97 \%$. In the case of L. monocytogenes, C. jejuni, and $S$.

772 aureus, the kmer length of 35 appears to be the optimal value to reach the same 
773 accuracy as mlst, which matches the original default and recommended parameter

774 value for stringMLST [17]. However, for S. enterica, a higher percentage of

775 agreement with MLST was achieved for kmer lengths of 55 and 65 (Fig 6). This

776 S. enterica-related observation recapitulated the initial findings of decreased

777 proportion of ST miscalls with higher kmer lengths (Fig 4C). Of note, our finding

778 collectively showed that the kmer length of 10 yielded low accuracy when

779 compared to mlst and other stringMLST kmer lengths. The most likely

780 explanation for lower accuracy generated by kmer 10 is that shorter kmers are

781 more likely to map unambiguously onto a genome when compared to other

782 lengths. That high frequency of kmer length 10 on a given dataset reflects their

783 higher likelihood of mapping to multiple regions of a genome (S1 Fig, S14A-B

784 Fig). Overall, stringMLST is a rapidly deployable and optimizable ST-based

785 genotyping algorithm that in this narrow-scope analysis proved to be applicable to

786 four phylogenetic distinct pathogens.

787

788 Optimization of stringMLST kmer length across

789 phylogenetic divergent species

790 Here, we systematically investigated what kmer length would give the fewest ST

791 miscalls (optimized length) with stringMLST across a broader array of

792 phylogenetic divergent pathogens. Given our previous results, we first fine-tunned

793 our investigation into the S. enterica population given the genetic and ecological

794 diversity across serovars. For that, we selected data from twenty-three S. enterica

795 zoonotic serovars and ran stringMLST with wide range of kmer lengths $(20,30$, 
$79635,40,45,50,55,60,65,70,80,90)$. Fig 7 A shows the core-genome phylogeny

797 mapping of the optimized kmer length across all twenty-three serovars along with

798 their corresponding percentage of ST miscalls. More detailed information on the

799 distribution of the percentage of ST miscalls for all used kmer lengths $(20,30,35$,

$80040,45,50,55,60,65,70,80,90)$ is shown on S15A Fig. As it can be seen on Fig

801 7A, many serovars (S. Anatum, $S$. Braenderup, $S$. Javiana, $S$. Mbandaka, $S$.

802 Montevideo, S. Oranienburg, S. Poona, S. Schwarzengrund, S. Senftenberg, $S$.

803 Typhimurium) have $0 \%$ of miscalls when the default kmer length 35 was used. $S$.

804 Infantis and S. Derby show the lowest percentage of ST miscalls (3\% and 2\%

805 respectively) with higher value of kmer, e.g., 90. Interestingly, S. Saintpaul

806 showed the highest percentage of ST miscalls when only considering the range of

807 kmer lengths used for the initial analyses (10-90). To investigate this further, we

808 ran stringMLST for $S$. Saintpaul with kmer lengths up to 240 (240 was chosen

809 because the maximum read length for the $S$. Saintpaul dataset is 250 base pairs or

810 nucleotides) (S15C-D Fig). As it can be seen on S15C Fig, the fewest ST miscalls

811 for $S$. Saintpaul were produced when kmer of length 140 was used (22\%). When

812 comparing the percentage of ST miscalls between mlst and stringMLST, mlst

813 outperformed stringMLST for the used datasets and range of kmer lengths (the

814 percentage of miscalls for mlst ranged from $\sim 0-1 \%$ across all serovars, while the

815 percentage of miscalls for stringMLST ranged from $\sim 0-85 \%$ depending on the

816 serovar and kmer length used, with $2 \%$ being the median across all combinations

817 of serovar and kmer length for stringMLST). In addition to the percentage of ST

818 miscalls, we calculated the percentage of ST agreement between mlst and 
819 stringMLST with the range of kmer lengths (S15B Fig). While for some serovars

820 this percentage is the highest when kmer with length 35 is used (e.g., S. Anatum,

$821 S$. Braenderup, S. Javiana, $S$. Mbandaka, $S$. Montevideo, $S$. Oranienburg, $S$.

822 Poona, S. Schwarzengrund, S. Senftenberg, S. Typhimurium), for other serovars

823 (e.g., S. Derby, S. Dublin, S. Enteritidis, S. Hadar, S. Heidelberg, S. Infantis, $S$.

824 Kentucky, S. Saintpaul) the percentage of ST agreement between the two

825 programs was higher with higher kmer lengths (the percentage of ST agreement

826 varied from $\sim 14-100 \%$ depending on the serovar and kmer length used, with $97 \%$

827 being the median across all combinations of serovar and kmer length).

828 In order to widen the scope of our phylogenetic-based analysis, we assessed the

829 percentage of ST miscalls across varying kmer lengths for divergent bacterial

830 pathogens with Public Health relevance. We selected 14 distinct organisms and

831 ran stringMLST with wide range of kmer lengths $(20,30,35,45,55,65,70,80$,

832 90). Fig 7B depicts the $16 \mathrm{~S}$ rRNA-based phylogeny overlaid with the optimal

833 kmer length that minimized the percentage of ST miscalls. Of note, the phylogeny

834 contained fourteen distinct pathogens and twenty-three genomes across each

835 serovar of S. enterica. The distribution of the percentage of ST miscalls for all

836 used kmer lengths $(20,30,35,40,45,50,55,60,65,70,80,90)$ is shown on

837 S15E Fig. While the percentage of ST miscalls varied between $0 \%$ and $22 \%$

838 across the S. enterica serovars as shown in Fig 7A, the percentage of miscalls was

839 expectedly more variable for the fourteen bacterial pathogens, ranging from $1.2 \%$

840 to $74.9 \%$. The datasets for A. baumannii, C. jejuni, H. influenzae, K. pneumoniae,

841 L. monocytogenes, $N$. gonorrhoeae, S. aureus and S. pneumoniae showed the 
842 lowest percentage of ST calls with the default kmer length of 35. C. difficile and

843 M. tuberculosis had minimized ST miscalls with kmer lengths of 20 and 30

844 respectively, while $P$. aeruginosa with kmer length of 65. Interestingly, for $E$.

845 faecium and H. pylori, the optimal kmer lengths were 35 and 20, even though the

846 percentage of miscalls was high (74.9\% and 67.6\%). To further investigate this,

847 we ran stringMLST for E. faecium and H. pylori with kmer lengths up to 140 (140

848 was chosen because the maximum read length for the two datasets is 150 base

849 pairs or nucleotides) (S15G-H Fig, S15K-L Fig). As can be seen on the Figures,

850 the percentage of miscalls was higher with higher kmer lengths, and the lower

851 kmer lengths yielded fewer miscalls, albeit this number was still considerably

852 high. Additionally, we ran stringMLST on another set of randomly selected 100

853 paired-end reads for E. faecium (S15M-N Fig), H. pylori (S15I-J Fig) and

854 Enterococcus faecalis (S15O-P Fig). These 100 reads were not part of the initial

855 datasets and were chosen to validate that the initial random data selection was not

856 completely biased. We also added E. faecalis here due to its close phylogenetic

857 association with E. faecium. For E. faecium and H. pylori we observed the same

858 pattern with 100 reads as with 1,000 reads. On the other hand, the pattern for $E$.

859 faecalis was quite opposite with lowest percentage of ST miscalls of $5.43 \%$ for

860 kmer 35. When comparing the percentage of ST miscalls between mlst and

861 stringMLST, for some datasets, such as C. jejuni, H. pylori, L. monocytogenes, $M$.

862 tuberculosis, $N$. gonorrhoeae, S. aureus, mlst performed worse than stringMLST

863 (with increase in percentage of miscalls by $48 \%, 13 \%, 11 \%, 50 \%, 9 \%, 45 \%$

864 respectively). In addition to the percentage of ST miscalls, we calculated the 
865 percentage of ST agreement between mlst and stringMLST with the range of

866 kmer lengths (S15F Fig). Of note, in the case of stringMLST, when the optimal

867 kmer length was above the default parameter of 35, the ultimately selected kmer

868 length was picked based on our empirical evidence for longer kmers being

869 capable of speeding up the computational analysis.

870 In summary, while the default kmer length of 35 used by stringMLST performs

871 accurately across many organisms, our systems-based approach encompassed the

872 analysis of a broad array of phylogenetic divergent organisms revealed: i) intra-

873 and inter-species variation in the percentage of ST miscalls requires fine-tunning

874 of the kmer length parameter; ii) lack of association between taxonomy or

875 phylogenetic placement of organisms and the optimal kmer length; and iii) unique

876 species behave as outliers for which stringMLST cannot be directly applied with

877 the default settings.

878

\section{Incorporating stringMLST in ProkEvo}

880 ProkEvo was recently developed as an automated and scalable computational

881 platform for bacterial population genomics analyses that uses the Pegasus

882 Workflow Management System (WMS) [31]. In particular, ProkEvo facilitates

883 the use of a hierarchical approach for population stratification with different

884 layers of genotypic resolution. MLST-based classification of genomes into STs is

885 part of this hierarchical approach that has been proven to be predictive of

886 ecological traits such as AMR in S. enterica lineages [57]. However, ProkEvo

887 currently only uses the standard mlst algorithm for ST calls [21]. As part of this 
888 paper, the stringMLST program was incorporated into ProkEvo without any

889 disruption in its workflow. The workflow design of ProkEvo with both mlst and

890 stringMLST is shown on S16 Fig.

891 In order to compare the performance of ProkEvo with mlst and stringMLST,

892 randomly shuffled subsets derived from the original datasets used for C. jejuni, L.

893 monocytogenes, $S$. Typhimurium, and $S$. aureus were used. One random subset

894 contained 100 genomes, while the second one had 1,000 genomes. ProkEvo was

895 run using either mlst or stringMLST on Crane, one of the high-performance

896 computing clusters at the Holland Computing Center [23]. For mlst, the pipeline

897 used was previously established and included a few required steps, such as quality

898 trimming and adapter clipping, de novo assembly and assembly discarding prior

899 to the ST mapping [21]. Based on the inter-species results shown here (Fig 6), the

900 default kmer length of 35 was used with stringMLST for this comparison. The

901 outcomes measured for this analysis were: i) total running time (Fig 8A); ii) the

902 percentage of non-classified STs (Fig 8B); and iii) the percentage of agreement

903 between programs (Fig 8C).

904 While the runtime of using ProkEvo with mlst varied from $\sim 8$ to 34 hours for

905 the subset containing 100 genomes, the runtime of ProkEvo with stringMLST

906 varied from $\sim 25$ minutes to 3 hours (Fig 8 A). Similarly, for the larger datasets

907 containing 1,000 genomes, the runtime of ProkEvo with mlst varied from $\sim 17$ to

90839 hours, while the runtime of ProkEvo with stringMLST varied from $\sim 4$ to 8

909 hours. Regardless of the pathogen species tested, stringMLST speeded up the

910 analyses $\sim 4$ times when utilizing 1,000 genomes across species. 
911 In terms of accuracy in ST classifications, the use of stringMLST considerably

912 decreased the number of non-classified STs, regardless of the dataset size (100 or

913 1,000 genomes) and bacterial species (Fig 8B). In accordance, stringMLST

914 resulted in a higher frequency of genomes classified as novel STs (ST numbers

915 that were not classified by mlst) (S17 Fig). Additionally, the overall concordance

916 between mlst and stringMLST varies from $82 \%$ to $100 \%$ across all datasets. The

917 percentage of agreement is the lowest for $S$. Typhimurium, while it is the highest

918 for S. aureus (Fig 8C). The lower proportion of ST miscalls and high percentage

919 of agreement between programs for S. aureus, compared to other species, is

920 associated with its higher degree of genetic homogeneity (fewer dominant STs)

921 (S5 Fig). This difference in miscalls and concordance between programs may be

922 further explained by the variation in database sizes, since the PubMLST schemes

923 used for mlst have fewer alleles across all seven loci which results in fewer STs

924 compared to stringMLST as shown on S3 Table.

925 Previously, the scalability of ProkEvo was assessed by a comparative analysis

926 of its computational performance on Crane and OSG, using two datasets with

9272,392 and 23,045 genomes each (10 X difference), and the standard mlst approach

928 for ST calling [21]. To further demonstrate the gain in computational runtime

929 obtained with the use of stringMLST within ProkEvo, the complete $S$.

930 Typhimurium dataset containing 23,045 genomes was run on OSG. While

931 ProkEvo with mlst finished all ST calls in 26 days and 6 hours when OSG was

932 used as a computational platform [21], ProkEvo with stringMLST completed the

933 task in 3 days and 6 hours. Altogether, stringMLST provides fine-tunable and 
934 rapid alternative to mlst for scalable ST genotyping that is portable to be

935 implemented in any high-performance and high-throughput platform, with its use

936 being further facilitated by its implementation in ProkEvo.

937

\section{Discussion}

939 The incorporation of WGS technology has advanced the study of bacterial

940 populations, enabling new strategies of inferring traits associated with populations

941 at different scales of resolution that may be contributing to important ecological

942 characteristics and/or epidemiological patterns [21][32][33][34][35][36][37][38].

943 In particular, the use of a hierarchical population structure analysis permits

944 inference into ancestral vs. recent evolutionary relationships and consequently,

945 the patterns of genomic and phenotypic diversification among genotypic units can

946 be studied within these evolutionary contexts $[21][39][40][41][12][42][43]$.

947 ST-based classification is an integral part of the hierarchical genotyping

948 approach [21][27]. ST lineages are inferred from the patterns of allelic variation at

949 a relatively small (typically seven) set of conserved loci [8][9][44] and remain a

950 very useful context in which to study bacterial populations. However, as the field

951 transitions to WGS data, the ability to infer STs is bottlenecked by the

952 computationally intensive step of genome assembly. Thus, there is a great need

953 for algorithms that can efficiently infer STs from large WGS-based datasets.

$954[21][27][8][9][44]$ There are multiple tools available for MLST classification,

955 such as mlst [22], ARIBA Error! Reference source not found., stringMLST 
956 [17], MentaLiST Error! Reference source not found., STing Error! Reference

957 source not found.. In general, the available tools can be categorized based on the

958 input data they use - some tools use raw Illumina paired-end sequence data, while

959 others use de novo assemblies [16]. Using raw sequence data for ST-based

960 classification has a tremendous advantage especially in pathogen surveillance,

961 since all the computationally demanding steps prior to the de novo assembly are

962 bypassed and the STs calls are made as the sequence reads are generated.

963 Specifically, mlst uses de novo genome assemblies as an input and performs

964 mapping in order to align sequences to pre-downloaded allelic files across all

965 target loci. ARIBA identifies AMR-associated genes, single nucleotide

966 polymorphisms, and ST calls using Illumina paired-end raw sequencing reads.

967 ARIBA clusters the raw reads by mapping them to genes, and then performs local

968 assembly within clusters to identify AMR genes and ST calls. On the other hand,

969 stringMLST and MentaLiST rely on kmer matching between raw sequence reads

970 and available ST schemes that allows for fast mapping and ST-based typing. In a

971 narrow setting with few bacterial species, both tools are shown to be accurate and

972 fast for standard MLST classification, while providing comparable accuracy with

973 MentaLiST albeit using less computational resources Error! Reference source

974 not found.. STing is the successor of stringMLST - it uses the same algorithmic

975 approach with additional computational applications for large MLST schemes

976 such as ribosomal MLST (rMLST) and core-genome MLST (cgMLST) Error!

977 Reference source not found.. All these tools have integrated ST schemes and/or

978 provide utilities for downloading the available PubMLST databases. There are a 
979 few available comparisons of assembly-free tools for ST classification, and the

980 published studies are primarily focused on the computational resources used and

981 the percentage of correctly classified STs in relatively small data sets

$982[16][63][64]$. When tools were tested with real outbreak datasets $(L$.

983 monocytogenes, E. coli, C. jejuni, S. enterica) comprising 85 samples,

984 stringMLST showed the fastest running time of 80.8 minutes and high accuracy in

985 ST calls (100\%) [16]. While MentaLiST does not scale well when reads with high

986 coverage are used, it performs well on MLST schemes with up to a few thousand

987 genes and alleles, such as cgMLST ( $\sim 3,000$ genes) [63]. While most ST tools

988 perform satisfactorily, there are some relevant bottlenecks to be considered. For

989 example, some tools use out-of-date MLST databases that require manual

990 curation, and can directly affect the accuracy of ST calls, especially when mixed

991 and low coverage samples are used [16]. ST tools that are assembly and alignment

992 free, such as stringMLST, STing and MentaLiST, show quite a few advantages in

993 term of accuracy and efficiency that make them applicable for real-time molecular

994 epidemiology and surveillance. Thus, we chose stringMLST as a representative of

995 the kmer-based ST tools to perform a systems-based comparative analysis that

996 assess the computational and statistical efficacy of ST calls across divergent

997 pathogens in contrast to the legacy MLST approach.

998 As shown in our studies, the accuracy of stringMLST is affected by the species

999 being tested without any specific phylogenetic patterns. In particular, the choice

1000 of kmer length used directly impacts the proportion of ST miscalls across species,

1001 and in certain cases it may not be applied as designed even after parameter 
1002 tunning. It is likely that the varying accuracy reflects the different levels of

1003 population structuring, patterns of genome diversification, degree of repetitive

1004 sequences, degree of horizontal gene transfer (HGT), and relative abundances of

1005 mobile elements such as prophages and insertion sequences, etc.

$1006[12][41][68][69][70][71][72][73]$. A clear example in our study was $S$. enterica,

1007 for which the accuracy of stringMLST at different kmer lengths varied across

1008 ecologically distinct serovars that are known to have unique pan-genomic

1009 composition such as prophage content [12][71][74][75]. To the best of our

1010 knowledge, the currently available comparisons between ST tools have not

1011 considered any systematic approach for parameter tunning across phylogenetic

1012 divergent species known to vary in population structure [21][27][67].

1013 In evaluating genome-intrinsic and -extrinsic variables that could contribute to

1014 differences in accuracy between mlst and stringMLST, we found that species

1015 level variation in accuracy was mostly explained by the uniqueness of their

1016 genomic composition and number of contigs per genome. As genomic

1017 composition is an inheritable property of the bacterial species, subtype, and

1018 evolutionary history of specific populations, the association with algorithmic

1019 performance was somewhat expected [46][47]. However, the contribution of the

1020 number of contigs to performance implies that data from long-read sequencing

1021 platforms such as PacBio and Oxford Nanopore Technologies (ONT) may have a

1022 considerable effect on accuracy of programs such as stringMLST because these

1023 technologies produce reads with lower accuracy $(\sim 80-90 \%)$ that may inflate the

1024 number of false allelic calls at MLST loci, with the effect of artificially splitting 
1025 major STs into multiple sub-populations [48]Error! Reference source not

1026 found.Error! Reference source not found.. Therefore, while more work is

1027 needed in this field, current studies using hybrid assembly approaches of both

1028 Illumina short reads and ONT long reads Error! Reference source not found., as

1029 well as only polished ONT reads Error! Reference source not found. for

1030 performing ST-based classification may lead to promising, cost-effective results

1031 from these types of platforms. Hence, we expect that a combination of hybrid

1032 sequencing strategies with species and even subtype-specific tuning of programs

1033 such as stringMLST and STing, will facilitate real-time surveillance, prediction of

1034 STs and prediction of traits such as AMR that are found to associated with

1035 specific STs [14][49].

1036 While the kmer length of 35 is currently recommended as a default value of

1037 stringMLST, our systems-based approach demonstrated that for specific bacterial

1038 species it will result in increasing the frequency of ST miscalls which in turn may

1039 hinder epidemiological investigations. Across phylogenetic divergent pathogenic

1040 bacterial species, the optimal kmer length ranged from 20 to 140, regardless of

1041 their ancestral relationship or speciation pattern. The varying population structure,

1042 the pattern of genome diversification and architecture (e.g., impact of HGT), as

1043 well as sequence coverage may be some of the reasons underlying the observed

1044 statistics [12][41][68][69][70][71][72][73]. Although we hypothesize that longer

1045 sequence reads will help overcome this limitation, there is still a context-

1046 dependent consideration for parameter tunning and overall algorithmic

1047 implementation. Therefore, in the case of stringMLST, we suggest the following 
1048 actionable strategies to maximize its utilization, including: i) developers to

1049 consider implementing a pre-step that heuristically searches for the optimal kmer

1050 length (minimizes ST miscalls) in dataset-dependent fashion (sampling from the

1051 testing data), perhaps even by comparing with the standard MLST program as

1052 positive controls; and/or ii) researchers to run wide range of kmer lengths on a

1053 subset of the dataset in order to select the optimal kmer length that minimizes the

1054 percentage of ST miscalls. Given the speed and scalability of stringMLST, using

1055 multiple kmer lengths is not likely to add much overhead to the analyses, and this

1056 provides an empirical statistical approach for kmer selection and optimization of

1057 ST classifications. With this data-driven fine-tunning of the kmer length,

1058 stringMLST is a powerful program that can be efficiently and effectively used in

1059 microbiological and epidemiological laboratories.

1060 We recently developed ProkEvo, a freely available scalable platform for

1061 performing hierarchical-based bacterial population genomics analyses [21].

1062 ProkEvo: 1) uses the Pegasus Workflow Management System to ensure

1063 reproducibility, scalability, and modularity; 2) uses high-performance and high-

1064 throughput computational platforms; 3) automates and scales multitude of

1065 computational analyses of a few to tens of thousands of bacterial genomes; 4) can

1066 run many thousands of analyses concurrently if the computational resources are

1067 available; 5) is easily modifiable and expandable platform that can incorporate

1068 additional algorithmic steps and custom scripts. The initial implementation of ST-

1069 based classifications through ProkEvo, as part of a hierarchical genotyping

1070 strategy to map and track populations, was done using the assembly-dependent 
1071 MLST program [21][22]. Running mlst inside ProkEvo allows for parallelization

1072 of the genome assemblies (run per isolate or genome) which enhances scalability

1073 and facilitates the optimal use of computation resources. Theoretically, if there

1074 are $n$ isolates and $n$ cores available on the computational platform, ProkEvo can

1075 linearly utilize all resources and run all $n$ independent tasks simultaneously.

1076 Typically, ST-based classifications are time consuming because the mapping

1077 process is run sequentially in a set of genomes instead of running them

1078 independently. Thus, using modular and distributed platforms such

1079 as ProkEvo for performing ST-based genotyping provides great benefit, especially

1080 if additional features such as other hierarchical genotypes and pan-genomic

1081 mapping tools are part of the same platform [21]. As part of this work, we

1082 modified ProkEvo to not only offer the standard assembly-dependent MLST

1083 mapping approach, but it now contains stringMLST, and our tests showed a

1084 significant speed-up in runtime for datasets ranging from a few hundreds to tens

1085 of thousands of genomes. Moreover, we additionally added STing to ProkEvo, as

1086 an efficient successor of stringMLST

1087 (https://github.com/npavlovikj/MLST_stringMLST_analyses/tree/main/Prokevo_

1088 STing). To use ProkEvo with stringMLST or STing, the researcher only needs to

1089 provide a list of SRA identifications and run the submit script without any

1090 advanced experience in high-performance or high-throughput computing.

1091 Depending on the configuration set, ProkEvo can use locally downloaded

1092 sequence data or download the data from NCBI directly. The Pegasus Workflow

1093 Managements System that is used by ProkEvo automatically handles the 
1094 dependencies, as well as all the intermediate and final files. Thus, using platforms

1095 such as ProkEvo with fast tool for hierarchical genotyping, such as stringMLST,

1096 allows for robust and efficient population-based genomics analyses that facilitate:

1097 i) mapping and tracking of variants or lineages for epidemiological inquiries; ii)

1098 population structure analysis; and iii) ecological trait prediction using pan-

1099 genomic mapping to specific genotypes.

1100

\section{Conclusion}

1102 In conclusion, stringMLST largely proved to be an accurate, rapid, and scalable

1103 tool for ST-based classifications that could be deployed in microbiological

1104 laboratories and epidemiological agencies. However, our work clearly illustrates

1105 the need to optimize stringMLST across phylogenetic divergent species and

1106 populations of bacterial pathogens. Based on our results, we propose that the kmer

1107 length should be optimized in two ways on a case-by-case basis: 1) intrinsically

1108 by implementing a pre-step inside the algorithm to sample from the target data

1109 and select the optimal kmer length; or 2) by the user through a heuristic data

1110 mining approach to select the optimal kmer length prior to finalizing the ST calls.

1111 Also, by assessing genome-intrinsic and -extrinsic factors that could affect the

1112 stringMLST performance, our work suggests that longer sequence reads have the

1113 potential to improve its accuracy for specific bacterial species. Furthermore, the

1114 integration of stringMLST into ProkEvo allows users to take advantage of other

1115 hierarchical genotyping strategies, including pan-genomic mapping, which

1116 reproducibly facilitates ecological and epidemiological inquiries at scale. 
1117 Ultimately, this work emphasizes the importance of developing robust algorithmic

1118 tools for mining WGS data that can have direct implications for mapping and

1119 tracking of bacterial populations.

1120

\section{Acknowledgements}

1122 This work was completed by utilizing the Holland Computing Center of the

1123 University of Nebraska, which receives support from the Nebraska Research

1124 Initiative, and using resources provided by the Open Science Grid, which is

1125 supported by the National Science Foundation and the U.S. Department of

1126 Energy's Office of Science. This research used the Pegasus Workflow

1127 Management Software funded by the National Science Foundation under grant

1128 \#1664162. This publication made use of the PubMLST website

1129 (https://pubmlst.org/) developed by Keith Jolley (Jolley \& Maiden 2010, BMC

1130 Bioinformatics, 11:595) and sited at the University of Oxford. The development

1131 of that website was funded by the Wellcome Trust. We would like to greatly

1132 thank Mats Rynge for his extensive assistance and valuable suggestions while

1133 setting up and running ProkEvo on the Open Science Grid. We also thank Dr.

1134 Derek Weitzel and Karan Vahi for their technical support.

1135 


\section{References}

1137 [1] Bedford J, Farrar J, Ihekweazu C, Kang G, Koopmans M, Nkengasong J.

1138 A new twenty-first century science for effective epidemic response.

1139 Nature. 2019 Nov;575(7781):130-6.

1140

[2] Lewnard JA, Reingold AL. Emerging challenges and opportunities in infectious disease epidemiology. American journal of epidemiology. 2019

1142 May 1;188(5):873-82.

1143 [3] Armstrong GL, MacCannell DR, Taylor J, Carleton HA, Neuhaus EB, Bradbury RS, Posey JE, Gwinn M. Pathogen genomics in public health. New England Journal of Medicine. 2019 Dec 26;381(26):2569-80.

[4] Achtman M. How old are bacterial pathogens?. Proceedings of the Royal Society B: Biological Sciences. 2016 Aug 17;283(1836):20160990.

1148 [5] Selander RK, Musser JM, Caugant DA, Gilmour MN, Whittam TS. Jul 1;3(1):1-7.

1151 [6] Shapiro BJ. How clonal are bacteria over time?. Current opinion in microbiology. 2016 Jun 1;31:116-23.

[7] Smith JM, Smith NH, O'Rourke M, Spratt BG. How clonal are bacteria?. 15;90(10):4384-8.

[8] Maiden MC, Bygraves JA, Feil E, Morelli G, Russell JE, Urwin R, Zhang 
populations of pathogenic microorganisms. Proceedings of the National Academy of Sciences. 1998 Mar 17;95(6):3140-5.

[9] Maiden MC. Multilocus sequence typing of bacteria. Annu. Rev. Microbiol.. 2006 Oct 13;60:561-88.

1163 [10] Feil EJ, Li BC, Aanensen DM, Hanage WP, Spratt BG. eBURST: inferring patterns of evolutionary descent among clusters of related bacterial genotypes from multilocus sequence typing data. Journal of bacteriology. 2004 Mar 1;186(5):1518-30.

1167 [11] Croucher NJ, Harris SR, Grad YH, Hanage WP. Bacterial genomes in epidemiology—present and future. Philosophical Transactions of the Royal Society B: Biological Sciences. 2013 Mar 19;368(1614):20120202. the population structure of Salmonella. PLoS genetics. 2018 Apr 5;14(4):e1007261.

1173 [13] Achtman M, Wain J, Weill FX, Nair S, Zhou Z, Sangal V, Krauland MG, Hale JL, Harbottle H, Uesbeck A, Dougan G. Multilocus sequence typing as a replacement for serotyping in Salmonella enterica. PLoS Pathog. 2012 Jun 21;8(6):e1002776.

1177 [14] Břinda K, Callendrello A, Ma KC, MacFadden DR, Charalampous T, Lee typing. Nature microbiology. 2020 Mar;5(3):455-64. 
1181 [15] MacFadden DR, Coburn B, Břinda K, Corbeil A, Daneman N, Fisman D,

1182

1183

1184

1185

1186

1187

1188

1189

1190

1191

1192

1193

1194

1195

1196

1197

1198

1199

1200

1201

1202

1203
Lee RS, Lipsitch M, McGeer A, Melano RG, Mubareka S. Using genetic

distance from archived samples for the prediction of antibiotic resistance

in Escherichia coli. Antimicrobial agents and chemotherapy. 2020 Mar

9;64(5):e02417-19.

[16] Page AJ, Alikhan NF, Carleton HA, Seemann T, Keane JA, Katz LS.

Comparison of classical multi-locus sequence typing software for nextgeneration sequencing data. Microbial genomics. 2017 Aug;3(8).

[17] Gupta A, Jordan IK, Rishishwar L. stringMLST: a fast kmer based tool for multilocus sequence typing. Bioinformatics. 2017 Jan 1;33(1):119-21.

[18] Abebe E, Gugsa G, Ahmed M. Review on major food-borne zoonotic bacterial pathogens. Journal of tropical medicine. 2020 Jun 29;2020.

[19] Centers for Disease Control and Prevention. Foodborne germs and illnesses. Centers for Disease Control and Prevention. https://www. cdc. gov/foodsafety/foodborne-germs. html. 2016.

[20] Centers for Disease Control and Prevention. Individual Salmonella serotypes reports. Centers for Disease Control and Prevention.

https://www.cdc.gov/salmonella/reportspubs/salmonella-atlas/serotypereports.html. 2020.

[21] Pavlovikj N, Gomes-Neto JC, Deogun JS, Benson AK. ProkEvo: an automated, reproducible, and scalable framework for high-throughput bacterial population genomics analyses. PeerJ. 2021 May 21;9:e11376.

[22] Seemann T, mlst Github https://github.com/tseemann/mlst. 
1204 [23] HCC. 2008. Holland computing center | Nebraska.

1205 [24] Pordes R, Petravick D, Kramer B, Olson D, Livny M, Roy A, Avery P,

1206 Blackburn K, Wenaus T, Würthwein F+6 more. 2007. The open science

1207 grid. Journal of Physics: Conference Series 78:12057

1208 [25] Sfiligoi I, Bradley DC, Holzman B, Mhashilkar P, Padhi S, Wurthwein F.

12092009 . The pilot way to grid resources using glideinWMS.

1210 [26] Anaconda. 2012. Anaconda | The World's Most Popular Data Science

$1211 \quad$ Platform.

1212 [27] Zhou Z, Alikhan NF, Mohamed K, Achtman M, Agama Study Group. The

1213 user's guide to comparative genomics with EnteroBase. Three case

1214 studies: micro-clades within Salmonella enterica serovar Agama, ancient

1215 and modern populations of Yersinia pestis, and core genomic diversity of

$1216 \quad$ all Escherichia. Biorxiv. 2019 Jan 1:613554.

1217 [28] Feil EJ, Cooper JE, Grundmann H, Robinson DA, Enright MC, Berendt T,

1218 Peacock SJ, Smith JM, Murphy M, Spratt BG, Moore CE. How clonal is

1219 Staphylococcus aureus?. Journal of bacteriology. 2003 Jun

$1220 \quad 1 ; 185(11): 3307-16$.

1221 [29] Oksanen J, Blanchet FG, Kindt R, Legendre P, Minchin PR, O'hara RB, 1222 Simpson GL, Solymos P, Stevens MH, Wagner H, Oksanen MJ. Package

1223 'vegan'. Community ecology package, version. 2013 Dec 12;2(9):1-295.

1224 [30] Jolley KA, Bray JE, Maiden MC. Open-access bacterial population

1225 genomics: BIGSdb software, the PubMLST. org website and their

1226 applications. Wellcome open research. 2018;3. 
1227 [31] Deelman E, Singh G, Su MH, Blythe J, Gil Y, Kesselman C, Mehta G,

1228

1229

1230

1231

1232

1233

1234

1235

1236

1237

1238

1239

1240

1241

1242

1243

1244

1245

1246

1247

1248
Vahi K, Berriman GB, Good J, Laity A. Pegasus: A framework for

mapping complex scientific workflows onto distributed systems. Scientific

Programming. 2005 Jan 1;13(3):219-37.

[32] Burnett E, Ishida M, De Janon S, Naushad S, Duceppe MO, Gao R, Jardim A, Chen JC, Tagg KA, Ogunremi D, Vinueza-Burgos C. Wholegenome sequencing reveals the presence of the blactx-m-65 gene in extended-spectrum $\beta$-lactamase-producing and multi-drug-resistant clones of salmonella serovar infantis isolated from broiler chicken environments in the Galapagos Islands. Antibiotics. 2021 Mar;10(3):267.

[33] Cooper AL, Low AJ, Koziol AG, Thomas MC, Leclair D, Tamber S, Wong A, Blais BW, Carrillo CD. Systematic evaluation of whole genome sequence-based predictions of Salmonella serotype and antimicrobial resistance. Frontiers in microbiology. 2020 Apr 3;11:549.

[34] Dallman TJ, Byrne L, Ashton PM, Cowley LA, Perry NT, Adak G, Petrovska L, Ellis RJ, Elson R, Underwood A, Green J. Whole-genome sequencing for national surveillance of Shiga toxin-producing Escherichia coli O157. Clinical Infectious Diseases. 2015 Aug 1;61(3):305-12.

[35] Pightling AW, Pettengill JB, Luo Y, Baugher JD, Rand H, Strain E. Interpreting whole-genome sequence analyses of foodborne bacteria for regulatory applications and outbreak investigations. Frontiers in microbiology. 2018 Jul 10;9:1482. 
1249 [36] Sheppard SK, Jolley KA, Maiden MC. A gene-by-gene approach to 1250 bacterial population genomics: whole genome MLST of Campylobacter.

1251 Genes. 2012 Jun;3(2):261-77.

1252 [37] Sheppard SK, Didelot X, Meric G, Torralbo A, Jolley KA, Kelly DJ, 1253 Bentley SD, Maiden MC, Parkhill J, Falush D. Genome-wide association study identifies vitamin B5 biosynthesis as a host specificity factor in Campylobacter. Proceedings of the national academy of sciences. $2013 \mathrm{Jul}$ 16;110(29):11923-7.

1257

1258

1259

1260

1261

1262

1263

1264

1265

1266

1267

1268

1269

1270

1271
[38] Alba P, Leekitcharoenphon P, Carfora V, Amoruso R, Cordaro G, Di Matteo P, Ianzano A, Iurescia M, Diaconu EL, Pedersen SK, Guerra B. Molecular epidemiology of Salmonella Infantis in Europe: insights into the success of the bacterial host and its parasitic pESI-like megaplasmid. Microbial genomics. 2020 May;6(5).

[39] Cheng L, Connor TR, Sirén J, Aanensen DM, Corander J. Hierarchical and spatially explicit clustering of DNA sequences with BAPS software. Molecular biology and evolution. 2013 Feb 13;30(5):1224-8.

[40] Tonkin-Hill G, Lees JA, Bentley SD, Frost SD, Corander J. Fast hierarchical Bayesian analysis of population structure. Nucleic Acids Research. 2019 Jun 20;47(11):5539-49.

[41] Didelot X, Bowden R, Street T, Golubchik T, Spencer C, McVean G, Sangal V, Anjum MF, Achtman M, Falush D, Donnelly P. Recombination and population structure in Salmonella enterica. PLoS genetics. 2011 Jul 28;7(7):e1002191. 
1272 [42] Gymoese P, Kiil K, Torpdahl M, Østerlund MT, Sørensen G, Olsen JE,

1273

1274

1275

1276

1277

1278

1279

1280

1281

1282

1283

1284

1285

1286

1287

1288

1289

1290

1291

1292

1293

1294
Nielsen EM, Litrup E. WGS based study of the population structure of

Salmonella enterica serovar Infantis. BMC genomics. 2019 Dec;20(1):1-1.

[43] Liao J, Orsi RH, Carroll LM, Wiedmann M. Comparative genomics

reveals different population structures associated with host and geographic

origin in antimicrobial-resistant Salmonella enterica. Environmental

microbiology. $2020 \mathrm{Jul} ; 22(7): 2811-28$.

[44] Maiden MC, Van Rensburg MJ, Bray JE, Earle SG, Ford SA, Jolley KA,

McCarthy ND. MLST revisited: the gene-by-gene approach to bacterial

genomics. Nature Reviews Microbiology. 2013 Oct;11(10):728-36.

[45] Spratt BG, Hanage WP, Li B, Aanensen DM, Feil EJ. Displaying the

relatedness among isolates of bacterial species-the eBURST approach.

FEMS microbiology letters. 2004 Dec 1;241(2):129-34.

[46] Bobay LM, Ochman H. Impact of recombination on the base composition of bacteria and archaea. Molecular biology and evolution. 2017 Oct

$$
1 ; 34(10): 2627-36 .
$$

[47] Raghavan R, Kelkar YD, Ochman H. A selective force favoring increased G + C content in bacterial genes. Proceedings of the National Academy of Sciences. 2012 Sep 4;109(36):14504-7.

[48] De Maio N, Shaw LP, Hubbard A, George S, Sanderson ND, Swann J, Wick R, AbuOun M, Stubberfield E, Hoosdally SJ, Crook DW.

Comparison of long-read sequencing technologies in the hybrid assembly of complex bacterial genomes. Microbial genomics. 2019 Sep;5(9). 
1295 [49] Page AJ, Keane JA. Rapid multi-locus sequence typing direct from

1296 uncorrected long reads using Krocus. PeerJ. 2018 Jul 31;6:e5233.

1297 [50] Bolger AM, Lohse M, Usadel B. Trimmomatic: a flexible trimmer for

1298 Illumina sequence data. Bioinformatics. 2014 Aug 1;30(15):2114-20.

1299 [51] Andrews S. FASTQC: a quality control tool for high throughput sequence 1300 data. Available at

1301 http://www.bioinformatics.babraham.ac.uk/projects/fastqc/. 2010.

1302 [52] Bankevich A, Nurk S, Antipov D, Gurevich AA, Dvorkin M, Kulikov AS, Lesin VM, Nikolenko SI, Pham S, Prjibelski AD, Pyshkin AV. SPAdes: a new genome assembly algorithm and its applications to single-cell sequencing. Journal of computational biology. 2012 May 1;19(5):455-77.

[53] Gurevich A, Saveliev V, Vyahhi N, Tesler G. QUAST: quality assessment tool for genome assemblies. Bioinformatics. 2013 Apr 15;29(8):1072-5.

[54] Treangen TJ, Sommer DD, Angly FE, Koren S, Pop M. Next generation sequence assembly with AMOS. Current Protocols in Bioinformatics. 2011 Mar;33(1):11-8.

[55] Rice P, Longden I, Bleasby A. EMBOSS: the European molecular biology open software suite. Trends in genetics. 2000 Jun 1;16(6):276-7.

1313 [56] Rizk G, Lavenier D, Chikhi R. DSK: k-mer counting with very low memory usage. Bioinformatics. 2013 Mar 1;29(5):652-3. 
genomes as a platform to enhance food safety. Front. Sustain. Food Syst.

[58] Valieris R. Parallel-fastq-dump. GitHub. Available at https://github.com/rvalieris/parallelfastq-dump. 2020.

Rang FJ, Kloosterman WP, de Ridder J. From squiggle to basepair: computational approaches for improving nanopore sequencing read accuracy. Genome biology. 2018 Dec;19(1):1-1.

1325 [60] Rhoads A, Au KF. PacBio sequencing and its applications. Genomics, proteomics \& bioinformatics. 2015 Oct 1;13(5):278-89.

\section{7 [61] Chen Z, Erickson DL, Meng J. Benchmarking hybrid assembly} approaches for genomic analyses of bacterial pathogens using Illumina and Oxford Nanopore sequencing. BMC genomics. 2020 Dec;21(1):1-21. numbers of samples. Microbial genomics. 2020 Mar;6(3). genomics. 2018 Feb;4(2).

1337 [64] Espitia-Navarro HF, Chande AT, Nagar SD, Smith H, Jordan IK, 
1340 [65] Hunt M, Mather AE, Sánchez-Busó L, Page AJ, Parkhill J, Keane JA, Harris SR. ARIBA: rapid antimicrobial resistance genotyping directly from sequencing reads. Microbial genomics. 2017 Oct;3(10).

1343 [66] Page AJ, Cummins CA, Hunt M, Wong VK, Reuter S, Holden MT, Fookes M, Falush D, Keane JA, Parkhill J. Roary: rapid large-scale prokaryote pan genome analysis. Bioinformatics. 2015 Nov $15 ; 31(22): 3691-3$.

[67] Zhou Z, Alikhan NF, Mohamed K, Fan Y, Achtman M, Brown D, Yersinia pestis phylogeny, and Escherichia core genomic diversity. Genome research. 2020 Jan 1;30(1):138-52. host species. Molecular ecology. 2011 Aug;20(16):3484-90. Molecular ecology. 2014 May;23(10):2442-51.

1360 [70] Yahara K, Didelot X, Ansari MA, Sheppard SK, Falush D. Efficient biology and evolution. 2014 Jun 1;31(6):1593-605. 
1363 [71] Mottawea W, Duceppe MO, Dupras AA, Usongo V, Jeukens J, Freschi L, Emond-Rheault JG, Hamel J, Kukavica-Ibrulj I, Boyle B, Gill A. Salmonella enterica prophage sequence profiles reflect genome diversity and can be used for high discrimination subtyping. Frontiers in microbiology. 2018 May 4;9:836.

1368 [72] den Bakker HC, Desjardins CA, Griggs AD, Peters JE, Zeng Q, Young SK, Kodira CD, Yandava C, Hepburn TA, Haas BJ, Birren BW. Evolutionary dynamics of the accessory genome of Listeria monocytogenes. PLoS One. 2013 Jun 25;8(6):e67511.

1372 [73] Castillo-Ramírez S, Corander J, Marttinen P, Aldeljawi M, Hanage WP, Westh H, Boye K, Gulay Z, Bentley SD, Parkhill J, Holden MT. Phylogeographic variation in recombination rates within a global clone of methicillin-resistant Staphylococcus aureus. Genome biology. 2012 Dec;13(12):1-3.

[74] Laing CR, Whiteside MD, Gannon VP. Pan-genome analyses of the species Salmonella enterica, and identification of genomic markers predictive for species, subspecies, and serovar. Frontiers in microbiology. 2017 Jul 31;8:1345.

1381 [75] Ferrari RG, Rosario DK, Cunha-Neto A, Mano SB, Figueiredo EE, Conte1382 Junior CA. Worldwide epidemiology of Salmonella serovars in animal1383 based foods: a meta-analysis. Applied and environmental microbiology. 2019 Jul 1;85(14):e00591-19. 
1385 [76] Price MN, Dehal PS, Arkin AP. FastTree: computing large minimum

1386 evolution trees with profiles instead of a distance matrix. Molecular

1387 biology and evolution. 2009 Jul 1;26(7):1641-50.

1388 [77] Letunic I, Bork P. Interactive Tree Of Life (iTOL) v5: an online tool for

1389 phylogenetic tree display and annotation. Nucleic acids research. 2021 Jul

$1390 \quad$ 2;49(W1):W293-6.

1391 [78] Seemann T. Prokka: rapid prokaryotic genome annotation. Bioinformatics.

$1392 \quad 2014$ Jul 15;30(14):2068-9.

1393 [79] Selander RK, Caugant DA, Ochman H, Musser JM, Gilmour MN,

Whittam TS. Methods of multilocus enzyme electrophoresis for bacterial

1395

population genetics and systematics. Applied and environmental

1396 microbiology. 1986 May;51(5):873-84.

1397 [80] Maslow JN, Whittam TS, Gilks CF, Wilson RA, Mulligan ME, Adams KS, Arbeit RD. Clonal relationships among bloodstream isolates of Escherichia coli. Infection and Immunity. 1995 Jul;63(7):2409-17.

1400 [81] Turner KM, Hanage WP, Fraser C, Connor TR, Spratt BG. Assessing the reliability of eBURST using simulated populations with known ancestry. BMC microbiology. 2007 Dec;7(1):1-4.

1403 [82] Chang Q, Abuelaish I, Biber A, Jaber H, Callendrello A, Andam CP, 1404 Regev-Yochay G, Hanage WP, PICR Study Group. Genomic epidemiology of meticillin-resistant Staphylococcus aureus ST22 widespread in communities of the Gaza Strip, 2009. Eurosurveillance.

$1407 \quad 2018$ Aug 23;23(34):1700592. 
bioRxiv preprint doi: https://doi.org/10.1101/2021.10.28.466354; this version posted November 16,2021 . The copyright holder for this preprint (which was not certified by peer review) is the author/funder, who has granted bioRxiv a license to display the preprint in perpetuity. It is made available under aCC-BY-NC-ND 4.0 International license.

1408 [83] Chang Q, Stevenson AE, Croucher NJ, Lee GM, Pelton SI, Lipsitch M,

1409 Finkelstein JA, Hanage WP. Stability of the pneumococcal population

1410 structure in Massachusetts as PCV13 was introduced. BMC infectious

1411 diseases. 2015 Dec;15(1):1-7. 


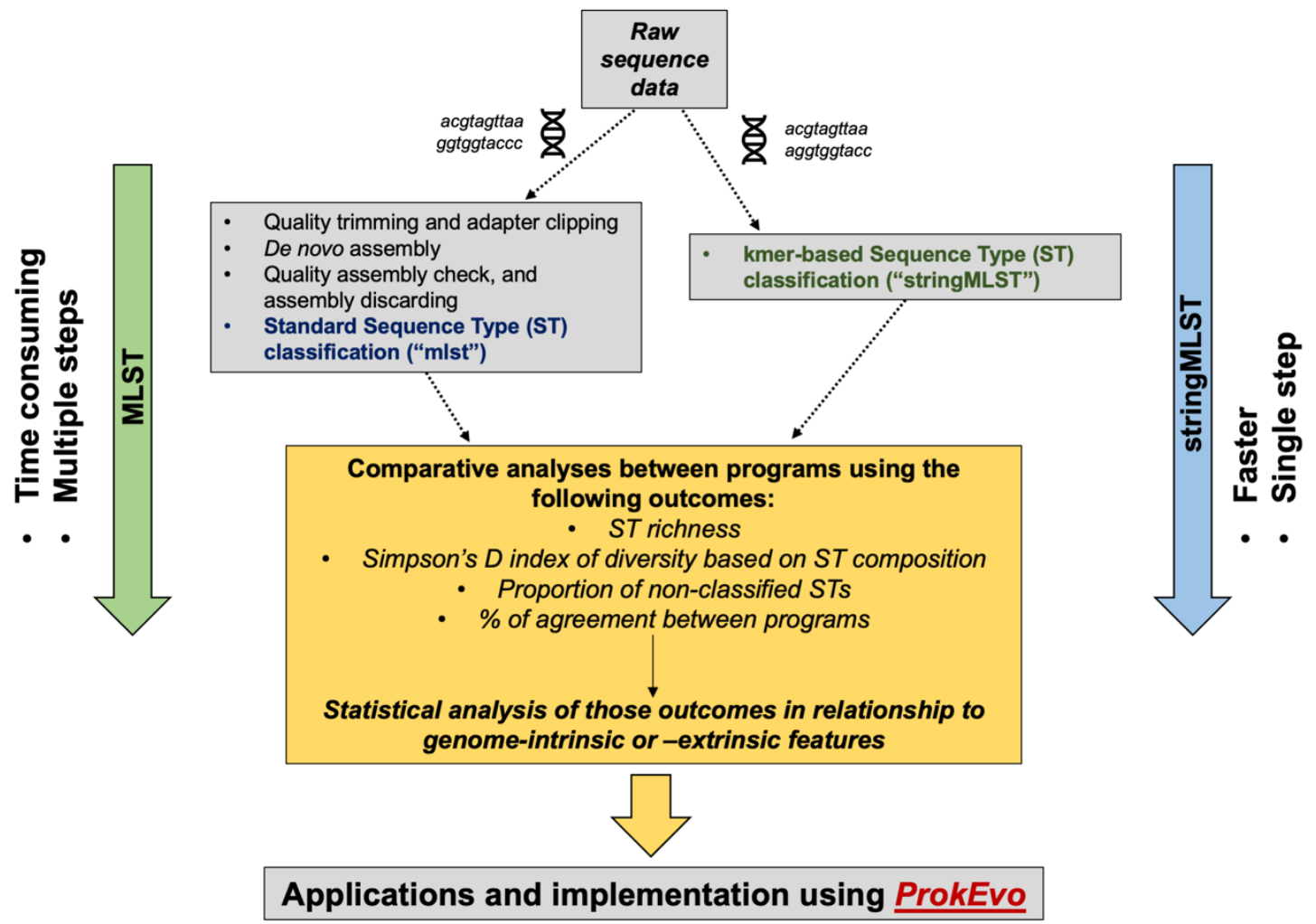

Fig 1. Computational workflow describing the analytical steps for a comparative analysis of two algorithms used for ST-based classification.

From top-down, the first step (narrow-scope) of the analytical approach entailed the acquisition and processing of Illumina paired-end raw reads from four distinct pathogens ( $C$. jejuni, L. monocytogenes, $S$. enterica and $S$. aureus), through an assembly-dependent (mlst) or assembly-free (stringMLST) approach for ST-based classification. Next, a set of comparative analyses encompassing measuring the computational performance, statistical metrics, and modeling were used to assess the accuracy and efficiency of mlst vs. stringMLST. Additionally, the contribution of genome-intrinsic and -extrinsic variables were used to identify explanatory factors that could impact the algorithmic efficiency across phylogenetic divergent species. Upon identification of inter-species differences in the performance of stringMLST, a wide-scope analysis was done to assess its accuracy across an array of fifteen phylogenetic divergent pathogenic species of bacteria with Public Health relevance. Ultimately, stringMLST was added to the computational platform ProkEvo to facilitate ST-based classification at scale, as part of a hierarchicalbased approach for population genomic analyses. 


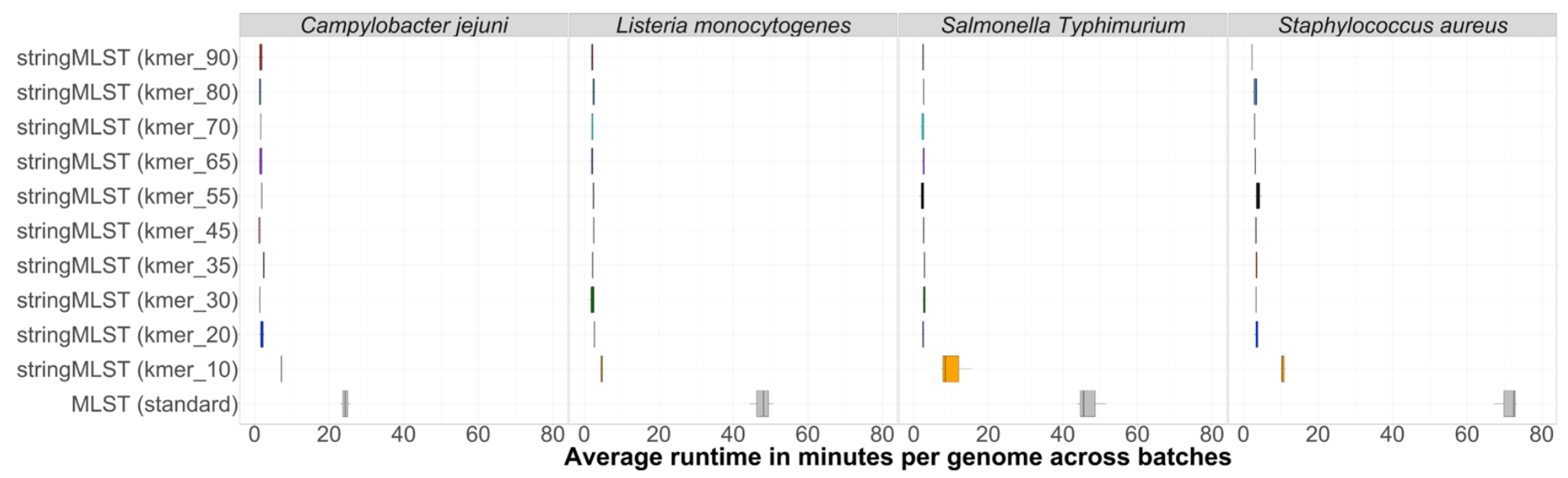

Fig 2. Box-and-whiskers plot showing the comparison of the average runtime per genome per batch (in minutes) needed by mlst and stringMLST for ST classification of genomes across four distinct bacterial species.

In order to compare the average runtime used by mlst and stringMLST with different kmer lenghts, we chose four different datasets, including four phylogenetic divergent bacterial pathogenic species: C. jejuni, L. monocytogenes, one major serovar of $S$. enterica (S. Typhimurium) and S. aureus - using 600 randomly selected genomes for each species. These 600 genomes were randomly split into three batches with 200 genomes each. We then ran mlst with all required steps, such as quality trimming and adapter clipping, de novo assembly and assembly discarding, on each batch and dataset. Separately, we ran stringMLST with a range of 10 different kmer values $(10,20,30,35,45,55,65,70,80,90)$ on each dataset, including the default length of 35 (y-axis). For each organism, the runtime was calculated as an average of 200 genomes per batch - since there were three batches, three datapoints were used to depict the distribution of runtime in minutes (x-axis). 
Factors that can influence ST-based classification using either MLST or stringMLST

\section{Genome-intrinsic variables}

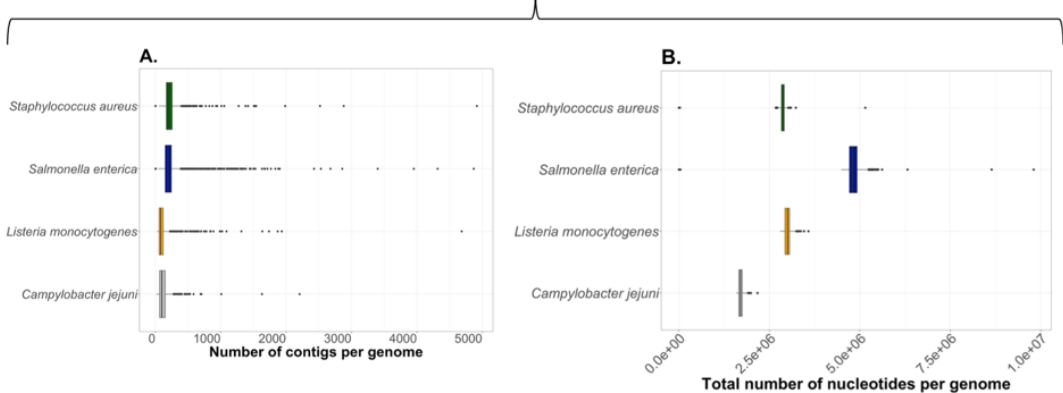

c.
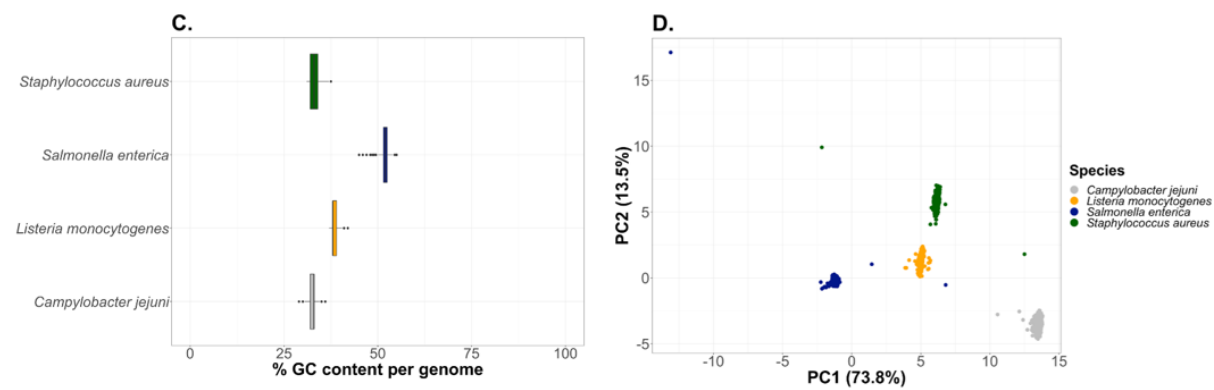
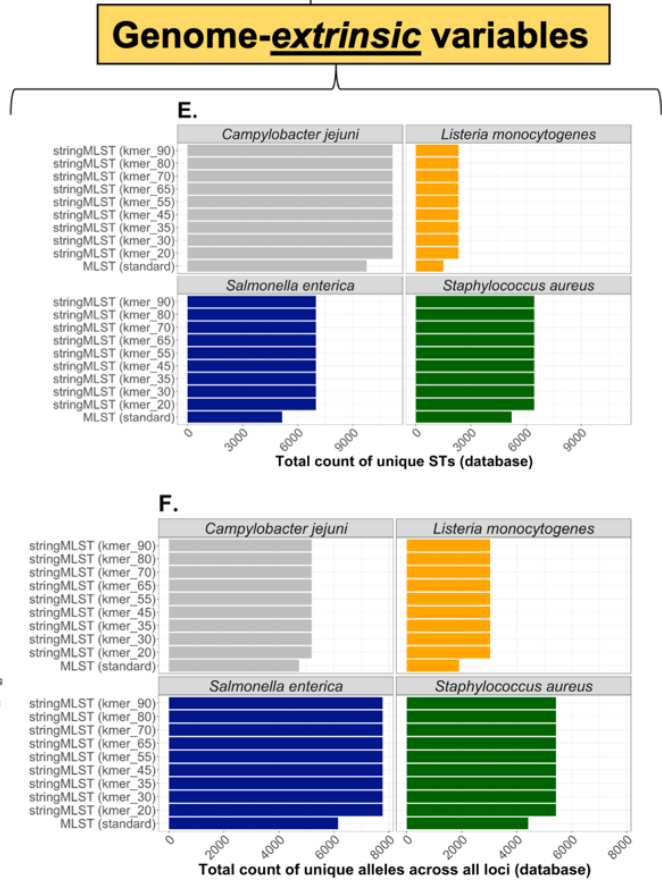

Fig 3. Genome-intrinsic and -extrinsic variables that can impact the accuracy of ST-based classification using either mlst (MLST-based genotyping) or stringMLST algorithmic approaches.

Box-and-whiskers plot showing genome-intrinsic variables, varying in distribution according to the bacterial species (A-C as y-axis), that may affect ST-based classification, include: (A) Number of contigs per genome (x-axis); (B) Total number of nucleotides per genome (x-axis); (C) GC\% content per genome (x-axis); and (D) Dinucleotide composition of genomes. (D) Inter-species PCA using the relative frequency of all pairs of dinucleotides (16 pairs) present in the genome as input data. Only two PCs are shown, and the percentage of variance explained by either PC is depicted in parenthesis. Bar plots showing genome-extrinsic variables that may influence the performance of mlst vs. stringMLST across species include but are not limited to: (E) Total count of unique STs per database (ST richness in the database used for mapping of raw reads or assemblies) (x-axis); and (F) Total count of unique alleles across all seven loci used for ST classification (x-axis). Specifically, the differences in ST richness and allelic composition in the databases reflect difference between mlst vs. stringMLST, and were not impacted by the kmer length (E-F, y-axis). 

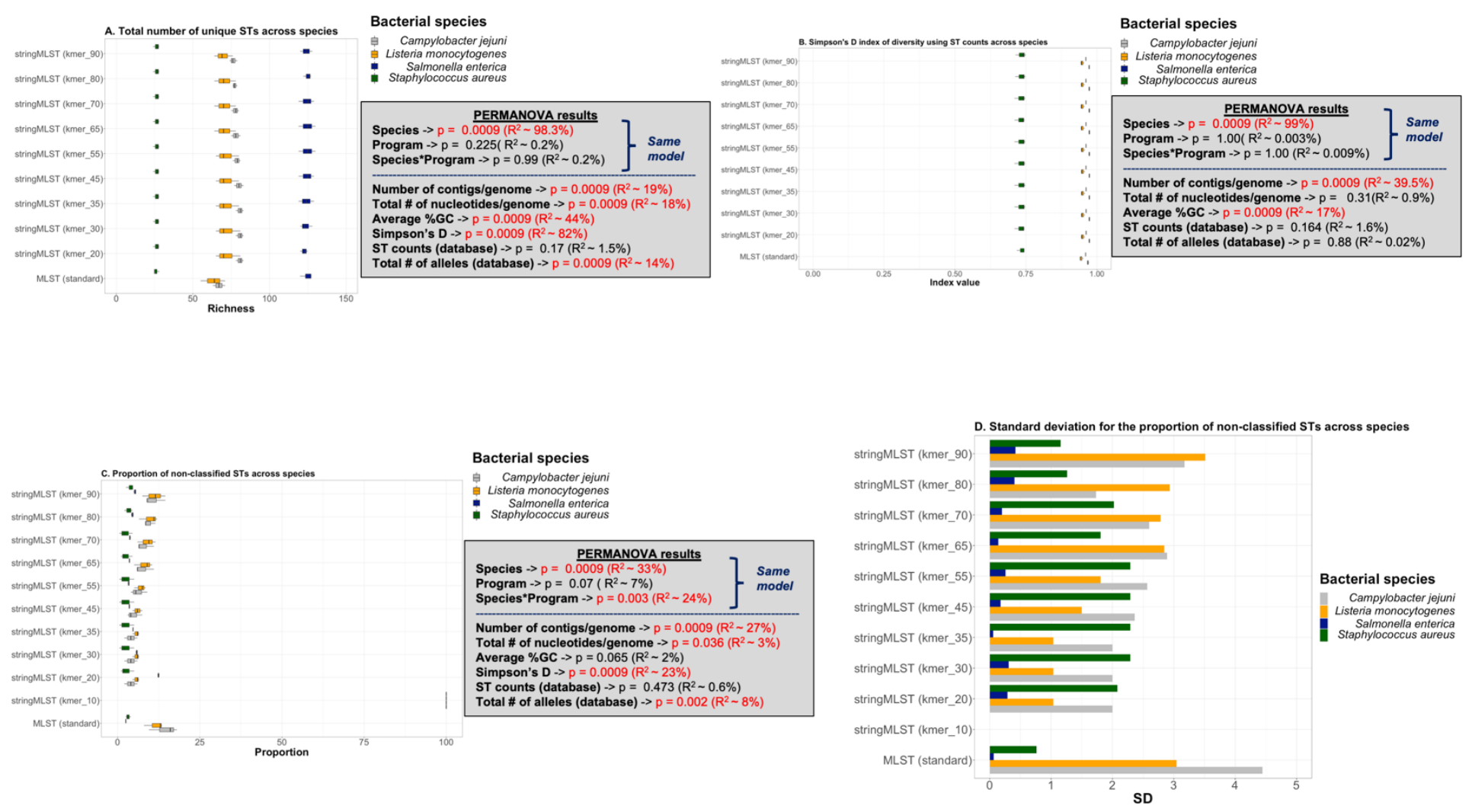

Fig 4. Statistical analysis of ST-based classification outcomes for comparison between mlst and stringMLST performance across bacterial species. (A-C) Box-and-whiskers plots A-C demonstrate the relationship between ST richness (x-axis), Simpson's index of diversity $(1-D)$ based on ST composition (x-axis), or the proportion of non-classified STs (x-axis) across bacterial species (color-coded differently) and programs (y-axis), respectively. Along with plots A$\mathrm{C}$ are depicted all PERMANOVA results including $p$-values $(p<0.05)$ and the univariate or synergistic contribution of factors measured by $R$-squared. PERMANOVA modeling was done in two specific ways: 1) A model including species, program, and their interaction, considering that those were the main variables of interest; and 2) All other results were calculated using univariate models and included modeling using genome-intrinsic (number of contigs per genome, total number of nucleotides per genome, and average $\mathrm{GC} \%$ content) and extrinsic (Simpson's D index of diversity, ST and allelic counts per database) variables. (D) Bar plot depicting the distribution of the standard deviation (SD, yaxis) for the proportion of non-classified STs based on species (color-coded differently) and programs (y-axis). 
A.

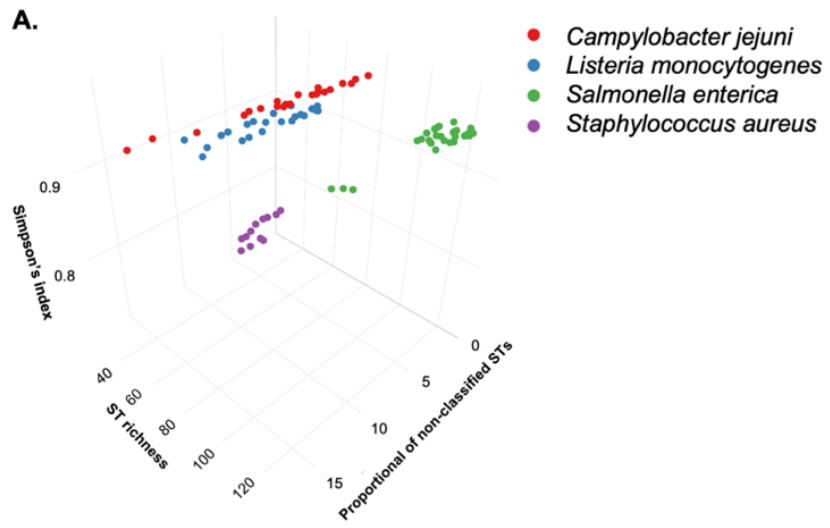

C.

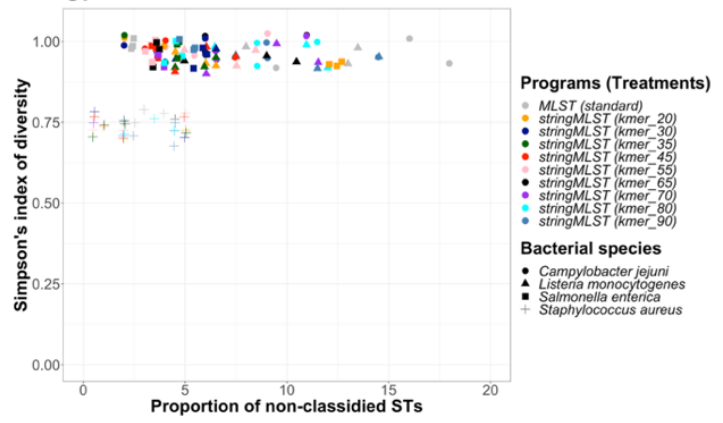

B.

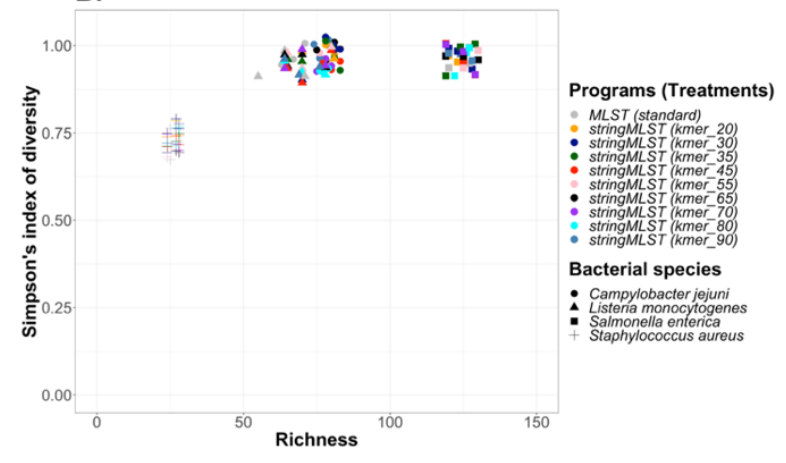

D.

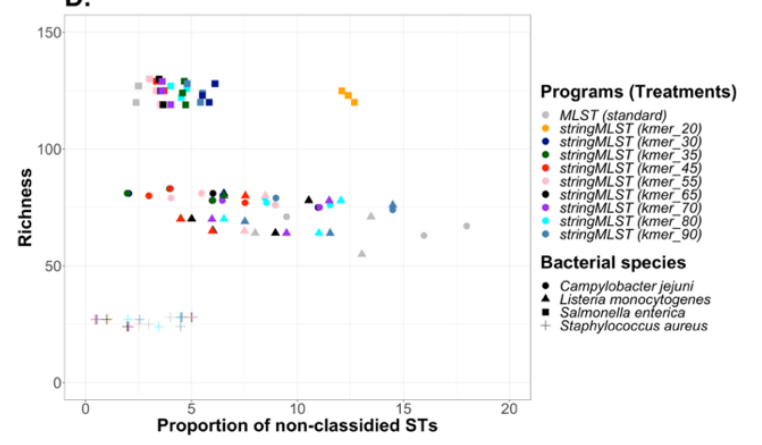

Fig 5. Multi-dimensional analysis of ST-based classification outcomes across different species using mlst vs. stringMLST.

(A) Tri-dimensional scatter plot demonstrating species grouping based on the outcomes calculated using the ST classification across programs, including: 1) Simpson's index of diversity (1 - D, Simpson's index); 2) ST richness; and 3) proportion of non-classified STs. (B-D) Biplots demonstrating groupings formed across species and programs based on the same outcomes. Scatter plot B depicts groupings produced based on the relationship between Simpson's index of diversity vs. ST richness (Richness); whereas scatter plot C shows the relationship between the Simpson's index of diversity and the proportion of non-classified STs; and lastly, scatter plot D depicts the relationship between ST richness (Richness) and the proportion of non-classified STs. 


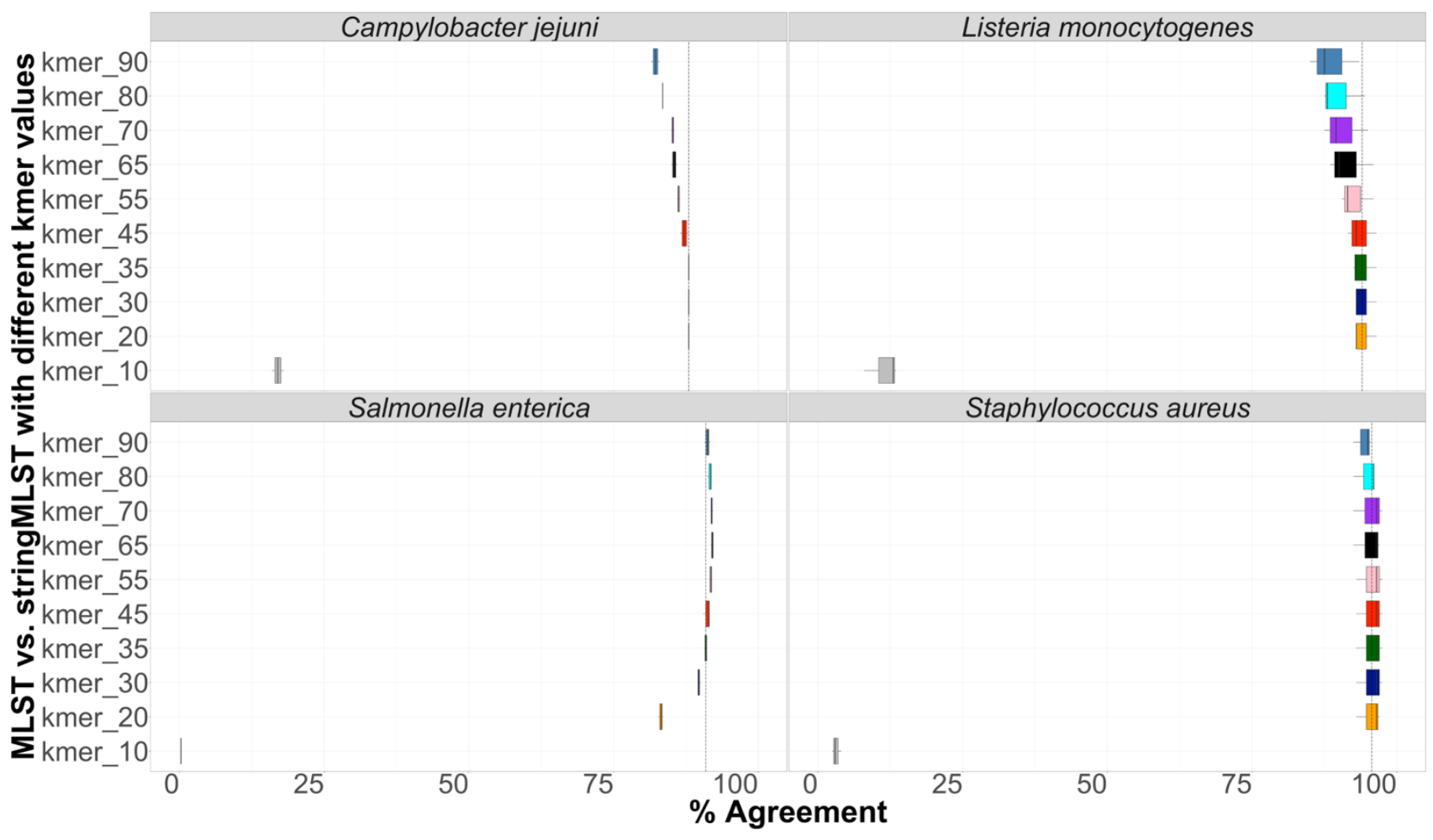

Fig 6. Box-and-whiskers plot depicting the concordance between mlst and stringMLST in ST calls.

Four different datasets belonging to four phylogenetic distinct bacterial pathogens, including $C$. jejuni (600 genomes), L. monocytogenes (600 genomes), $S$. enterica (11,787 genomes from 20 different serovars) and $S$. aureus (600 genomes) were run with mlst and stringMLST for ST-based classification. In the case of stringMLST, kmer lengths varied from 10 to 90 to identify the optimal value (highest percentage of agreement with the standard MLST approach), across all four species (y-axis). If both programs outputted identical ST calls (either number of missing/blank value), the call was defined as a match; otherwise, it was identified as a mismatch, and the percentage of agreement (x-axis, concordance) was calculated accordingly. The dashed line on the $\mathrm{x}$-axis represents the percentage agreement for the kmer value of 35 which is used as a default parameter by stringMLST. 


\section{A.}
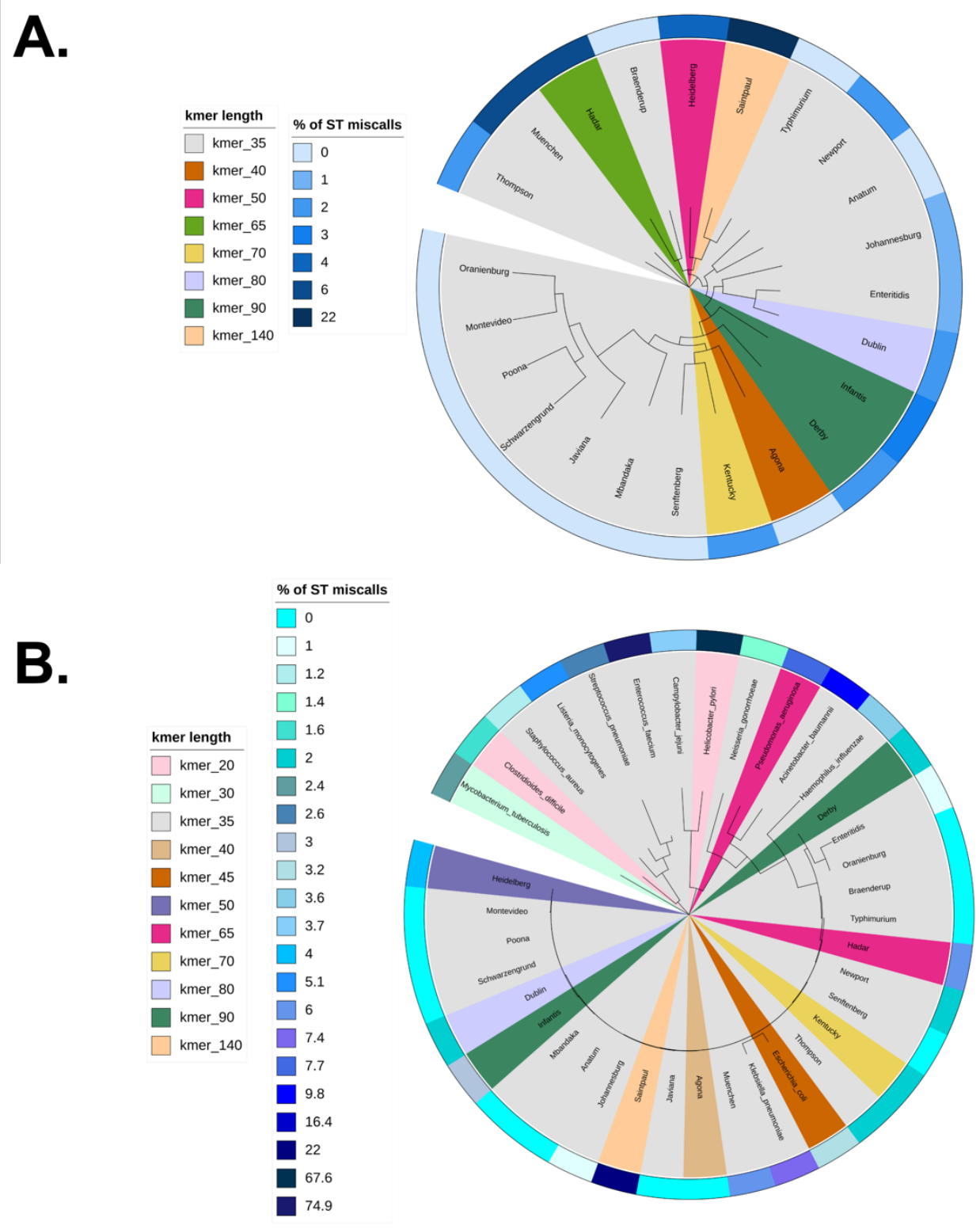

Fig 7. Phylogeny-guided display of optimal kmer length and algorithmic performance when using stringMLST for ST mapping across bacterial species. (A) Core-genome based phylogenetic display of stringMLST results across the twenty-three zoonotic serovars of Salmonella enterica subsp. enterica lineage I ( $S$. enterica). The branches are colored based on the optimal kmer length which gives the lowest percentage of ST miscalls (ST calls that returned missing/blank values for stringMLST). The outer ring present in the phylogeny is colored based on the corresponding ST miscall percentages associated with each optimal kmer length. The dataset used to identify the optimal kmer length and percentage of ST miscalls 
was composed of 2,300 genomes (100 genomes per serovar) and the phylogenetic tree was generated using twenty-three core-genome sequences (one of each serovar to facilitate data visualization); (B) Single locus-based (i.e., 16S rRNA gene) phylogenetic display of stringMLST results across fourteen divergent bacterial pathogens, including twenty-three representative genomes across each zoonotic serovar of the $S$. enterica species. The tree branches are colored based on the optimal kmer length which minimizes the percentage of ST miscalls (ST calls that returned missing/blank values for stringMLST). The outer ring present in the phylogeny corresponds to ST miscall percentage associated with each optimal kmer length. The dataset used to identify the optimal kmer length and percentage of ST miscalls was composed of 14,000 genomes (1,000 genomes for each bacterial pathogen) and 2,300 Salmonella genomes (100 genomes per serovar). The phylogeny (B) was generated using the $16 \mathrm{~S}$ rRNA representative sequences across 37 bacterial species (one for each species was used to facilitate visualization). All phylogeny-based visualization were generated using iTOL version 6.4 . 


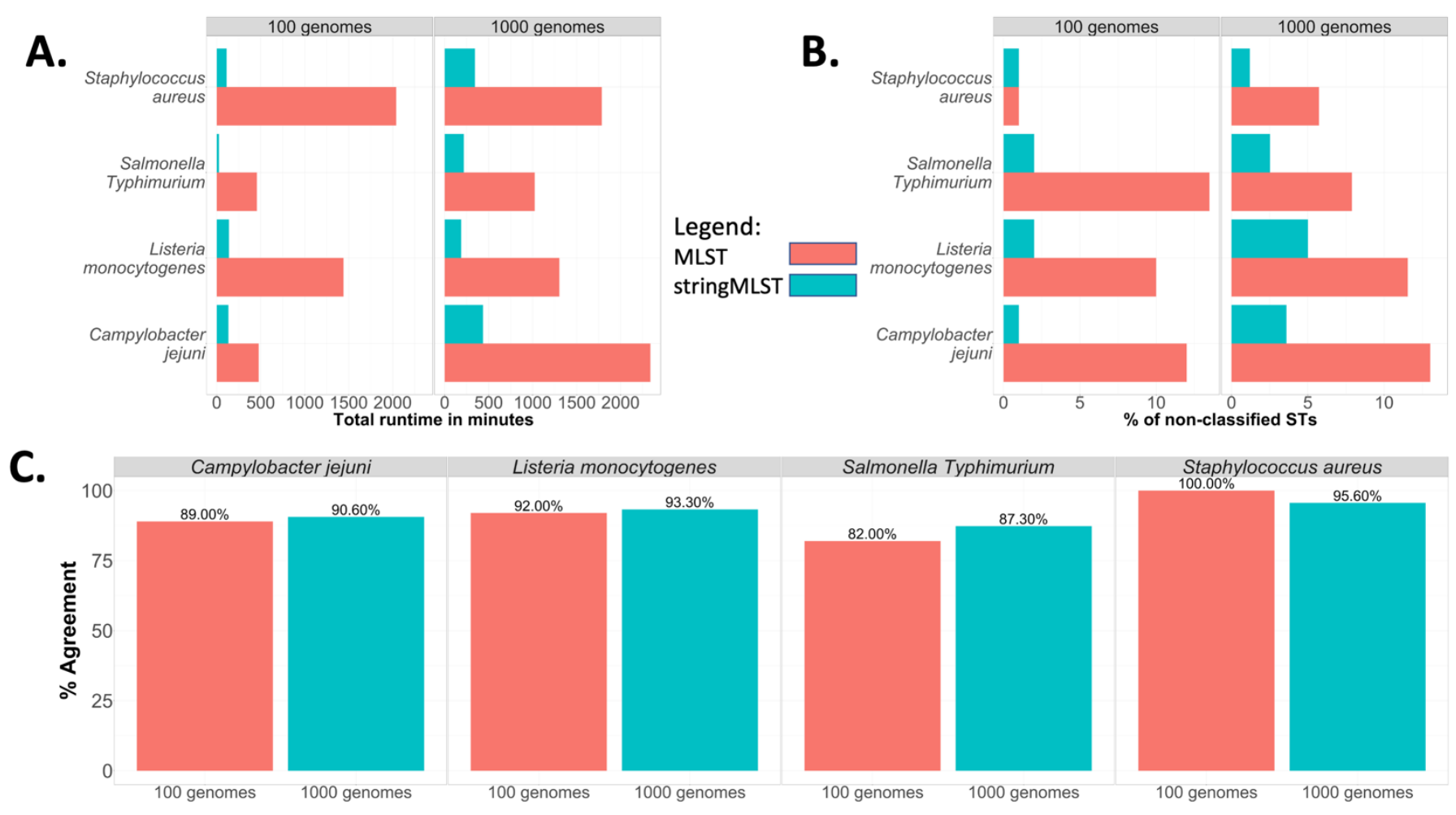

Fig 8. Comparison between the computational and statistical performance of mlst and stringMLST when using ProkEvo to run both programs.

Two subsets, one with 100 and the second one with 1,000 randomly chosen genomes, were selected from $C$. jejuni, L. monocytogenes, one major serovar of $S$. enterica ( $S$. Typhimurium) and $S$. aureus to compare the performance of running mlst or stringMLST through ProkEvo. The performance and statistical metrics used for comparison were: (A) Total runtime of individual workflow in minutes; (B) Percentage of non-classified STs (ST calls that returned missing/blank values); and (C) Percentage of agreement (concordance) between programs ("good" or "bad" ST calls that matched between mlst and stringMLST). 